\title{
Genome Analysis of Two Newly Emerged Potato Late Blight Isolates Sheds Light on Pathogen Adaptation and Provides Tools for Disease Management
}

\author{
Fan Zhang, ${ }^{1}$ Han Chen, ${ }^{1,2}$ Xinjie Zhang, ${ }^{1}$ Chuyun Gao, ${ }^{1}$ Jie Huang, ${ }^{1,2}$ Li Lü, ${ }^{1}$ Danyu Shen, ${ }^{1,2}$ Luyao Wang, ${ }^{3}$ Chong Huang, ${ }^{4}$ \\ Wenwu Ye, ${ }^{1,2}$ Xiaobo Zheng, ${ }^{1,2}$ Yuanchao Wang, ${ }^{1,2,5}$ Jack H. Vossen, ${ }^{6}$ and Suomeng Dong $1,2,5, \dagger$ \\ ${ }^{1}$ Department of Plant Pathology, Nanjing Agricultural University, Nanjing, 210095, China \\ ${ }^{2}$ Key Laboratory of Integrated Management of Crop Diseases and Pests, Ministry of Education, Nanjing, 210095, China \\ ${ }^{3}$ Agricultural Genomics Institute at Shenzhen, Chinese Academy of Agricultural Sciences, Guangdong Laboratory for Lingnan Modern \\ Agriculture, Shenzhen, 518120, China \\ ${ }^{4}$ National Agro-Tech Extension and Service Center, Maizidian Street, No. 20, Beijing, 100125, China \\ ${ }^{5}$ Key Laboratory of Plant Immunity, Nanjing Agricultural University, Nanjing, 210095, China \\ ${ }^{6}$ Plant Breeding, Wageningen University and Research, Wageningen 6708 PB, The Netherlands \\ Accepted for publication 2 October 2020.
}

ABSTRACT

\begin{abstract}
Phytophthora infestans, the causal agent of the Irish Potato Famine in the $1840 \mathrm{~s}$, is one of the most destructive crop pathogens that threaten global food security. Host resistance $(R)$ genes may help to control the disease, but recognition by through the gene products can be evaded by newly emerging isolates. Such isolates are dangerous as they may cause disease outbreaks under favorable conditions. However, our lack of knowledge about adaptation in these isolates jeopardizes an apt response to resistance breakdown. Here we performed genome and transcriptome sequencing of HB1501 and HN1602, two field isolates from distinct Chinese geographic regions. We found extensive polymorphisms in these isolates, including gene copy number variations, nucleotide polymorphisms, and gene expression changes. Effector encoding genes, which contribute to virulence, show distinct expression landscapes in P. infestans isolates HB1501 and
\end{abstract}

HN1602. In particular, polymorphisms at multiple effectors required for recognition (Avr loci) enabled these isolates to overcome corresponding $R$ gene based resistance. Although the isolates evolved multiple strategies to evade recognition, we experimentally verified that several $R$ genes such as $R 8, R B$, and Rpi-vnt1.1 remain effective against these isolates and are valuable to potato breeding in the future. In summary, rapid characterization of the adaptation in emerging field isolates through genomic tools inform rational agricultural management to prevent potential future epidemics.

Keywords: analytical and theoretical plant pathology, epidemics, genome, mycology, Phytophthora infestans, potato late blight, resistance, transcriptome
Potato late blight caused by Phytophthora infestans, was responsible for the Irish Potato Famine and remains a serious threat to global potato and tomato production (Birch et al. 2012). Deployment of resistance $(R)$ genes in agriculture is a promising method to manage this destructive oomycete pathogen in a sustainable way (Haverkort et al. 2016). Although $R$ genes have been introgressed into cultivated potato (Solanum tuberosum), P. infestans is able to overcome this resistance rapidly (Vleeshouwers et al. 2011).

The $P$. infestans genome counts nearly 240 megabases $(\mathrm{Mb})$ of which $74 \%$ consists of repetitive sequences, and it is the largest and most complicated genome sequenced among chromalveolates so far (Haas et al. 2009). This genome consists of conserved genedense regions (GDR) and gene-sparse regions (GSR). The repeat rich GSRs serve as sites of dynamic variation. Meanwhile, genes located in GSRs accumulate sequence polymorphisms and tend to display accelerated evolutionary signatures (Chen et al. 2018; Dong

${ }^{\dagger}$ Corresponding author: S. Dong; smdong@njau.edu.cn

Funding: This work is supported by Chinese National Science Funds (31972252 and 31721004), Postgraduate Research \& Practice Innovation Program of Jiangsu Province (020-Z562015603), and Fok Ying-Tong Education Foundation (151025).

*The $\boldsymbol{e}$-Xtra logo stands for "electronic extra" and indicates there are supplementary materials, supplementary tables, and a supplementary protocol published online.

The author(s) declare no conflict of interest.

This article is in the public domain and not copyrightable. It may be freely reprinted with customary crediting of the source. The American Phytopathological Society, 2021. et al. 2015). Furthermore, GSR gene expression is significantly regulated during infection (Qutob et al. 2013). Recent evidence suggests that genes occupying GSR display higher levels of adenine N6-methylation than genes occupying the GDR (Chen et al. 2018). These epigenetic signatures underlie phenotypic plasticity and rapid adaptation to environmental variation and host changes (Dong et al. 2015; Raffaele et al. 2010a).

Previous studies suggested that genes encoding effectors and epigenetic control enzymes are under selection during the evolution of Phytophthora species from Clade 1c (Raffaele et al. 2010a). A key factor in the successful infection and propagation of $P$. infestans in plants is a diverse set of effector proteins carrying the conserved RxLR (Arg-any-Lys-Arg) motif (Birch et al. 2009; Dou et al. 2008). It is known that plant species have evolved $R$ genes that encode nucleotide binding leucine rich repeat (NLR) proteins, which function as intracellular receptors to recognize a subset of RxLR effectors from Phytophthora species (Jones et al. 2016; Thilliez et al. 2019). The effectors that are recognized are referred to as avirulence (AVR) proteins. AVR proteins activate the host's effector-triggered immunity (ETI) and prevent pathogens from successful infection of plants (Chisholm et al. 2006; Jones and Dangl 2006).

Gene deletion, mutation, and silencing of Avr genes enable $P$. infestans to escape the recognition by the cognate $\mathrm{R}$ proteins. Evasion of recognition by R1, R2, R3b, and Rpi-blb1 were shown to correspond with gene deletions of Avr1, Avr2, Avr3b, and Avrblb1 in different $P$. infestans isolates (Vleeshouwers et al. 2011). Moreover, several examples of sequence polymorphisms in Avr loci are known to gain virulence. For example, isolates with $A V R 3 a^{\mathrm{EM}}$ instead of $A V R 3 a^{\mathrm{KI}}$ can evade recognition by $\mathrm{R} 3 \mathrm{a}$ 
(Armstrong et al. 2005). Avrblb2 is part of a highly polymorphic gene family, and the residue substitutions at position 69 was reported to evade Rpi-blb2 recognition (Oh et al. 2009; Oliva et al. 2015). The loss of recognition by $\mathrm{R} 4$ is due to a frameshift mutation in Avr4 resulting in a premature stop codon in virulent isolates (van Poppel et al. 2008). Besides sequence variations, transcriptional polymorphism of $A v r$ genes plays a role in pathogen adaptation on resistant plants. Avrvnt1 silencing in isolate P13626 from Ecuador results in evasion of recognition by Rpi-vnt1.1 (Pais et al. 2018).

There are continuous fluctuations in $P$. infestans populations all over the world (Martin et al. 2016; Yoshida et al. 2013). The deployment of potato varieties with $R$ gene mediated resistance has played positive roles in the prevention and control of late blight worldwide, but it also imposes huge selection pressures on P. infestans populations (Bradshaw et al. 2004). The virulence of $P$. infestans isolates is defined as their ability to overcome the resistance in a differential set of resistant varieties. In potato, the conventional differential set includes $R I$ through $R I l$ and was recently updated and expanded (Zhu et al. 2014). Recent studies demonstrated that $P$. infestans isolates have evolved more complicated virulence types as field populations have rapidly changed in several parts of the world. The EU13_A2 is an aggressive lineage that displaced most of the other lineages in the United Kingdom in a short time. Genome analyses of a EU13_A2 isolate revealed extensive genetic and expression polymorphisms particularly in effector genes (Cooke et al. 2012). These variations may contribute to resistance breakdown and aggressiveness, factors that drive field population displacement. In general, newly emerged isolates tend to overcome more $R$ genes simultaneously due to genetic or epigenetic changes occurring at Avr loci (Gijzen et al. 2014; Zhu et al. 2014).

Recently, we observed that newly emerged Chinese field isolates HB1501, from a north China population, and HN1602, from a south China population, both evade recognition by the R1 to R11 Stirling potato varieties. The frequency of so-called supervirulent isolates (overcoming R1 to R11 simultaneously) was slightly lower but rapidly increased from nearly 1 to $10 \%$ recently (Xiao-Hu et al. 2013). In addition, the Chinese isolate $\operatorname{Pd} 21410$ was reported as supervirulent in a north-western China population (Yin et al. 2017). However, the mechanisms by which these isolates evade $\mathrm{R}$ protein recognition remain unknown. In order to reveal such mechanisms, we investigated genome-wide variation and gene expression levels during infection with HB1501 and HN1602. We showed that isolates containing variations in the sequence or expression of $A v r$ genes were indeed able to evade recognition from R1, R3a, R3b, and $\mathrm{R} 4$, resulting in a failure to trigger plant immunity and allowing the onset of disease. Meanwhile, we found that HB1501 and HN1602 can be recognized by R8, Rpi-blb1, Rpi-blb2, and Rpi-vnt1.1. These results imply that $R$ genes can be rapidly and effectively deployed as prevention strategies to control epidemics of potato late blight.

\section{MATERIALS AND METHODS}

Plant growth and Phytophthora inoculation assay. $P$. infestans isolates were routinely cultured on solid rye sucrose agar (RSA) $+10 \% \mathrm{~V} 8$ medium at $18^{\circ} \mathrm{C}$ in the dark. Nonsporulating hyphae were cultured at $18^{\circ} \mathrm{C}$ in the dark using RSA $+10 \% \mathrm{~V} 8$ liquid medium for 6 to 7 days. Hyphae were collected and immediately frozen in liquid nitrogen. Potato cultivars Désirée and Stirling (R1 to R11) were used to provide leaves for detached leaflet assay after growing at $25^{\circ} \mathrm{C}(14 \mathrm{~h})$ and $20^{\circ} \mathrm{C}(10 \mathrm{~h})$ for 4 to 6 weeks.

The isolates in Supplementary Table S1 were collected by Yan Shu and Li Lü (Li et al. 2017a; Shu 2015), and these isolates were stored in liquid nitrogen for further processing. The mating type of each of the 11 isolates collected in this study was tested by pairing with known standard isolates (80029: A1 and 88133: A2) on rye A agar plates. The mating types of Phytophthora species, referred to as A1 and A2, do not signify the production of particular sex organs, as isolates of either mating type are hermaphroditic and thus capable of producing both antheridia and oogonia, the male and female reproductive organs, respectively (Judelson 1997). Each mating type secretes a specific hormone $(\alpha 1$ or $\alpha 2)$ that induces sexual reproduction in isolates of the opposite mating type (Harutyunyan et al. 2008), but the isolates of A1A2 mating type as self-fertile isolates can induced sexual reproduction with no other isolates (Zhu et al. 2016). Mating type of each isolate was determined by the sputum culture method (Qi et al. 2005). Sporangia of $P$. infestans isolates HB1501, HN1602, and 06_3928A were harvested in cold ultrapure water after growing on RSA $+10 \% \mathrm{~V} 8$ medium plates for 12 days at $18^{\circ} \mathrm{C}$, and zoospores were collected after 1 to $2 \mathrm{~h}$ of incubation at $4^{\circ} \mathrm{C}$. Potato leaves (cultivar Desiree) were inoculated with $10-\mu \mathrm{l}$ drop containing around 2,000 to 4,000 zoospore. The inoculation was applied onto abaxial sides of detached leaflets on wet paper towels. Two droplets per leaflet were applied for each of the two Chinese field isolates. A total of nine leaflets were used corresponding to 18 replicates per time point for each isolate.

To assess differential virulence of $P$. infestans HB1501 and HN1602 on isogenic potato plants, fully developed leaves of 4- to 6week-old isogenic potato varieties and wild-type 'Désirée' plants were detached and infected with zoospores, as described above. Inoculated leaves were incubated for 4 days under controlled environmental conditions $\left(18^{\circ} \mathrm{C}, 14 \mathrm{~h}\right.$ of light and $10 \mathrm{~h}$ of dark).

For RT-PCR experiments, 5 to $10 \mu \mathrm{g}$ of total RNA from infected leaf tissue and from mycelia was subject to DNAse treatment with the DNA-free Kit (Ambion). cDNA synthesis was performed with $500 \mathrm{ng}$ of DNAse-treated RNA using the PrimerScript RT reagent Kit (TaKaRa). cDNA (1 $\mu \mathrm{l})$ and genomic DNA (200 ng) samples were then used as template in PCRs using rTaq DNA polymerase (TaKaRa), following manufacturer's instructions. Primers used were listed in Supplementary Table S14 and tubulin as control. Presence/absence of amplification products was evaluated in 1 to $1.5 \%$ agarose gels, and the GeneRuler 5,000-bp DNA marker (TaKaRa) was used to assess amplicon size.

Whole-genome resequencing and sequence analyses. The Chinese isolates HB1501 and HN1602 were sequenced with the Illumina Hi-Seq 2000. The sequencing generated, on average, 100 million 150-bp paired-end reads for each isolate. The sequencing results were deposited in the Sequence Read Archive (SRA) at the NCBI database (accession number PRJNA604447).

The reference genome of isolate T30-4 and the resequencing data of various $P$. infestans isolates analyzed in this study were published in previous studies (Cooke et al. 2012; Li et al. 2017b; Yoshida et al. 2013). The clean Illumina reads of each isolate were mapped to the reference genome with Burrows-Wheeler transform alignment (BWA) software package v0.7.17 (Cooke et al. 2012), and single nucleotide polymorphism (SNP) calling was conducted subsequently, using samtools/bcftools and Sequence Alignment/ Maptools ( $\mathrm{Li}$ et al. 2017b). The resequenced isolates 06_3928A selected from previous studies were reanalyzed (Supplementary Table S2). Several criteria were considered in SNP filtering: (i) an SNP should be biallelic between each isolate and T30-4 genomes; (ii) at the SNP locus, the phred quality score of base sequencing and score of read mapping should both be higher than 10; (iii) each allele of an SNP should be supported by at least four reads; and (iv) the nonreference allele frequency should be between 0.2 and 0.8 at a heterozygous SNP locus (Yoshida et al. 2013). Furthermore, SNPs calling were optimized by bioinformatic parameters to reach $99.0 \%$ accuracy and $85.0 \%$ sensitivity with false discovery rate analysis with method in previous report (Cooke et al. 2012).

The phylogenetic tree was generated with genome sequence data. To test the genomic characterization of these two newly emerged isolates, genome resequencing data of HB1501 and HN1602 were analyzed with other previous reported $P$. infestans isolates ( $\mathrm{Li}$ et al. 2017b; Yoshida et al. 2013). Firstly, we called SNPs from published whole-genome sequence data and built predicted genome of each 
isolate by mapping SNPs to the reference genome. Then the reassembled scaffold 1.1 of each isolate was analyzed, as scaffold 1.1 is the largest scaffold in the reference genome, and the likelihood phylogenetic analysis tree was generated with genomelevel data instead of traditional simple sequence repeat markers. Homologous SNPs of scaffold 1.1 in 16 isolates were aligned to each other with MAFFT software package v7.427, and the alignment was multiplied with PhyML software package v3.1 (Martin et al. 2016). Evolutionary analyses were conducted in MEGA7, and the percentage of replicate trees in which the associated taxa clustered together in the bootstrap test $(1,000$ replicates) are shown next to the branches.

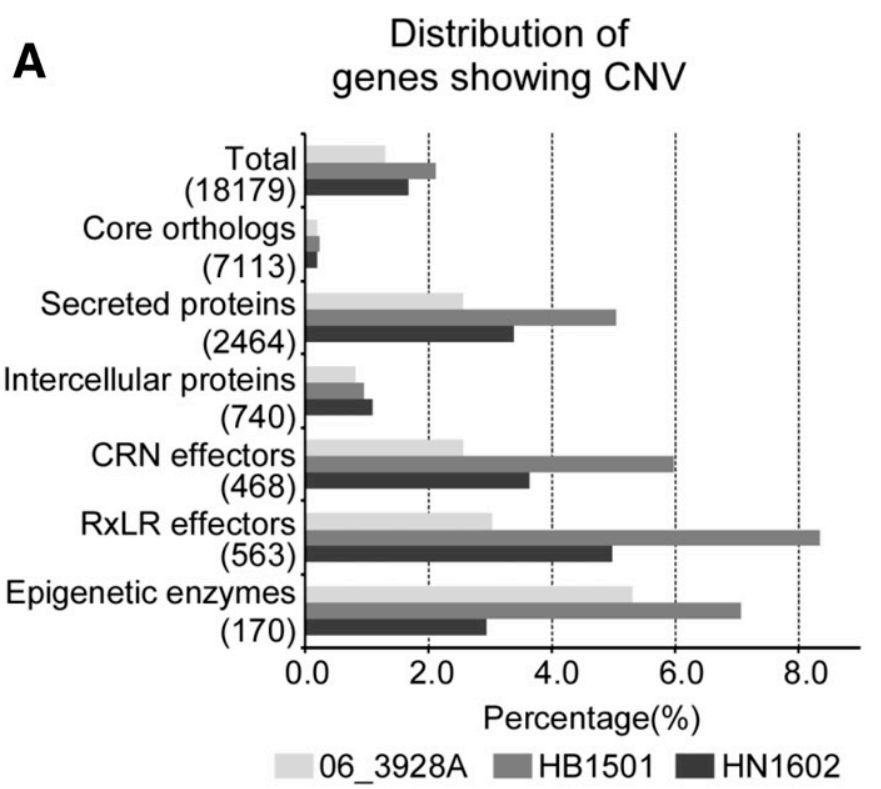

B

Ploidy was determined based on the mean frequency of heterozygous positions, with example for a single and diploid mode at 0.5 , while 0.33 and 0.67 were expected for triploid genomes. The SNPs of $P$. infestans HB1501 and HN1602 were aligned with different ploidy levels using methods described from previous studies (Li et al. 2017b).

For estimation of copy number, the average breadth of read coverage, read depth per gene, and gene copy numbers were calculated as described previously (Cooke et al. 2012). Genes with no coverage were considered absent. Copy numbers were estimated from the average read depth (ARD) as previously described. ARD for the coding sequence of a gene ' $\mathrm{g}$ ' (ARD [g]) was calculated and

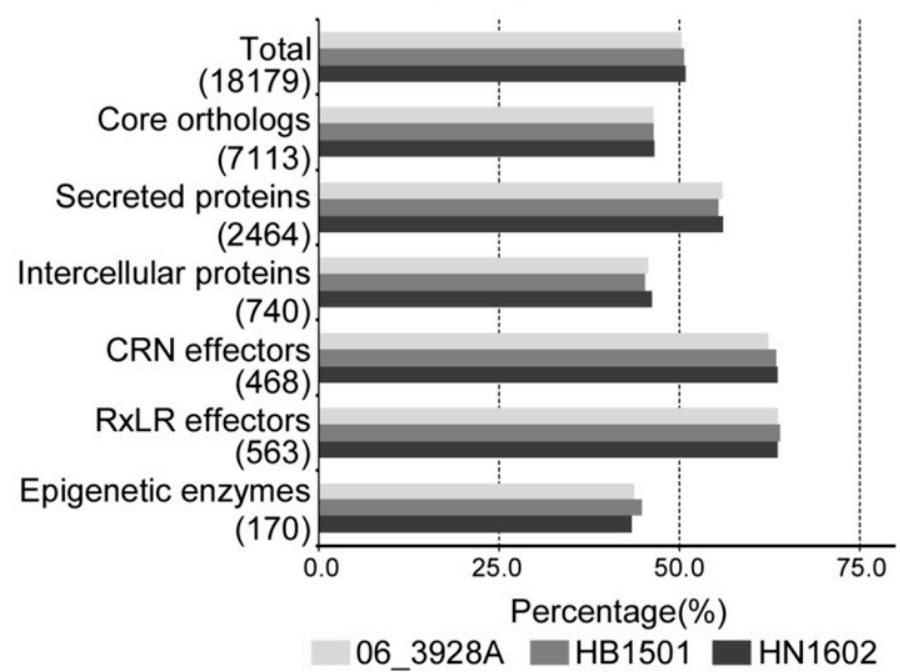

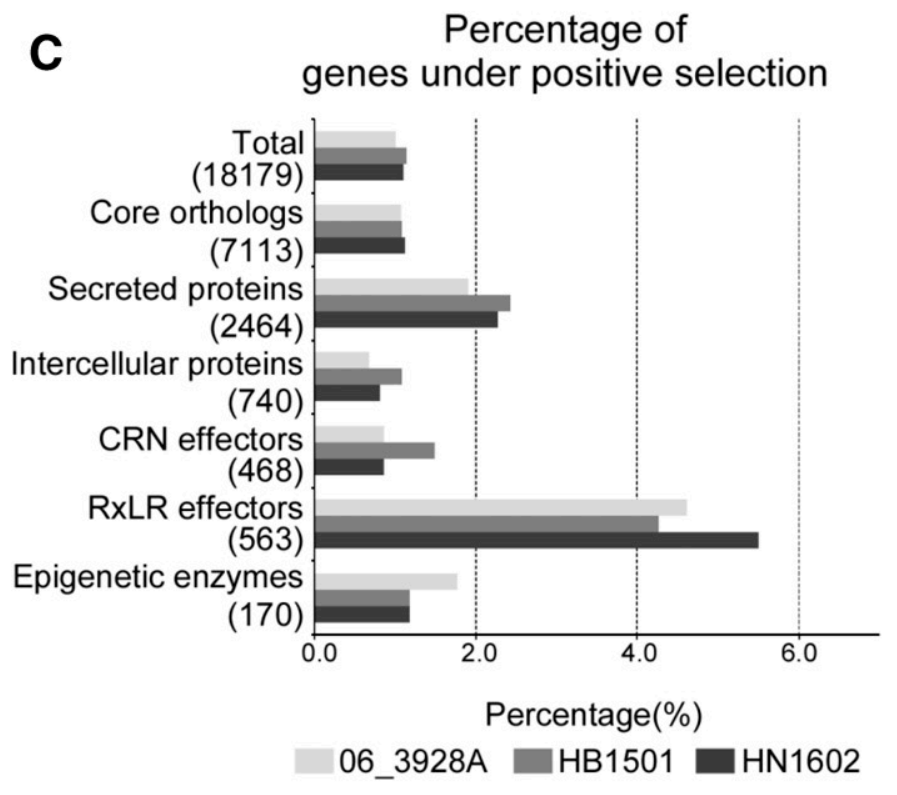

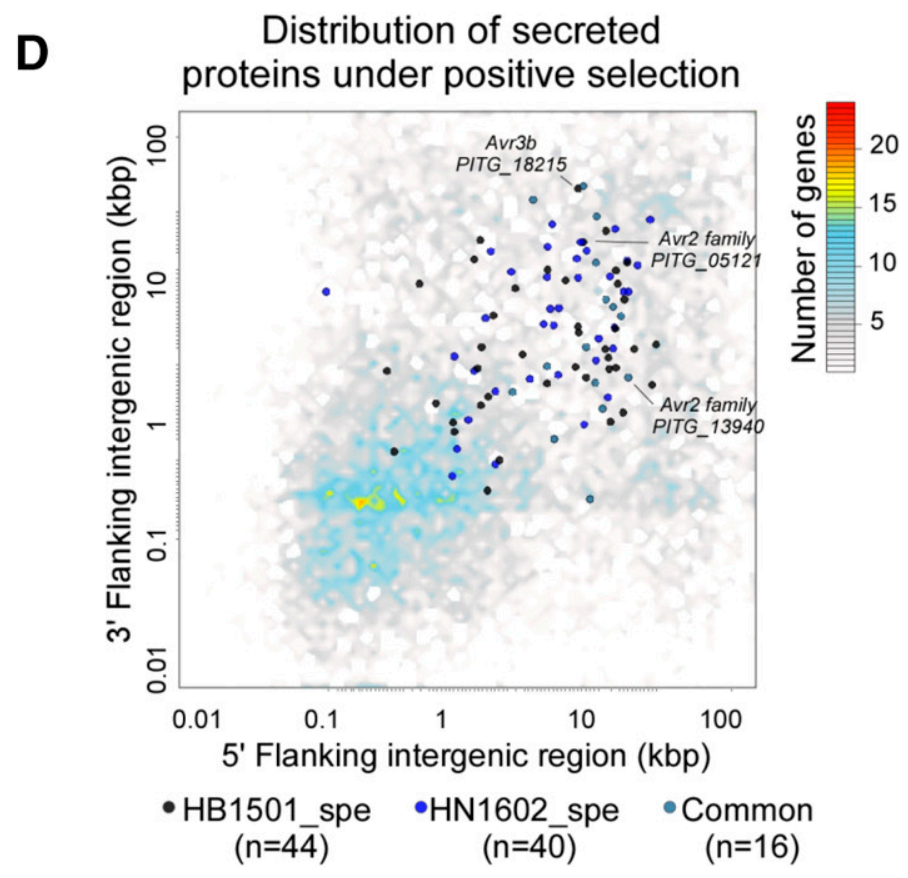

Fig. 1. Sequence polymorphism analysis of two nearly emerged isolates. $\mathbf{A}$ and $\mathbf{B}$, The distribution of genes showing copy number variations (CNVs) and nonsynonymous single nucleotide polymorphism (SNP) variations were calculated in Chinese isolates HB1501 and HN1602 and the U.K. isolate 06_3928A. T30-4 genome was referred to as control. C, Distributions of genes under positive selected genes $(\mathrm{dN} / \mathrm{dS}>1)$. D, The secreted genes under positive selection are highly enriched in gene sparse region in HB1501 and HN1602. Distribution of secreted genes in both isolates according to gene density (measured as the length of 5' and $3^{\prime}$ flanking intergenic regions). Three Avr genes are highlighted. n, the number of secreted genes in all three examined isolates. 
adjusted according to GC content. Adjusted ARD for a gene ' $\mathrm{g}$ ' (AARD [g]) belonging to the ith GC content percentile was obtained by the formula AARD $[\mathrm{g}]=\mathrm{ARD}[\mathrm{g}] \times \mathrm{mARD} / \mathrm{mARD}_{\mathrm{GC}}$, where $\mathrm{mARD}$ is the overall mean depth of all coding sequences and $\mathrm{mARD}_{\mathrm{GC}}$ is the mean depth for genes in the ith GC content percentile. Copy number for a gene ' $\mathrm{g}$ ' $(\mathrm{CN}[\mathrm{g}])$ was calculated as AARD $[\mathrm{g}] / \mathrm{mARD}$. Copy number variation for a gene ' $\mathrm{g}$ ' (CNV [g]) was calculated as $\mathrm{CN}[\mathrm{g}]$ in HB1501 and HN1602. Genes with the same $\mathrm{CN}$ in both isolates will have $\mathrm{CNV}[\mathrm{g}]=0$. Genes with $\mathrm{CNV}$ $[\mathrm{g}] \geq 1$ or $\mathrm{CNV}$ [g] $\leq-1$ were considered as having copy number polymorphisms between HB1501, HN1602, and 06_3928A.

Among proteins with signal peptides, a distinction can be made between intercellular proteins, RxLR effectors, CRN effectors, and other secreted proteins as identified in P. infestans before (Haas et al. 2009; Raffaele et al. 2010b). We sorted out intercellular proteins including cysteine proteases, lipases, serine carboxypeptidases, protease inhibitors, elicitin-like proteins, glycoside hydrolases, polysaccharide lyases, secreted serine proteases, and Nep1-like proteins as previously defined (Haas et al. 2009). Epigenetic proteins were sorted out with methods from previous studies (Chen et al. 2018; Wang et al. 2019). The heatmap of Figure 1D was performed with the reported method (Dong et al. 2015).

The pectate lyases of $P$. infestans were screened with pfam03211 and pfam00544. Then, the reported pectate lyases of $P$. capsici are used to characterize genes as PL1, PL3, and CE8 with BLASTP (Fu et al. 2015). For example, the annotation of PITG_08558 is pel05PL1, which means PITG_08558 is close to pel05 and belongs to the CAZY family PL1 (Supplementary Table S13).

Unmapped reads of each isolate were sorted with the method from a previous study (Cooke et al. 2012). Unmapped reads were assembled using the SOAPdenovo2 with a Kmer of 81, a minimum contig length of $200 \mathrm{bp}$ nucleotides, and insertion length of $300 \mathrm{bp}$ as parameters. All contigs of each isolate were analyzed with the NUCmer program, included in MUMmer software package v3.2. Signal peptides were predicted from predicted proteins using SignalP v5.0 and TMHMM programs. Secreted proteins were selected with the parameters as SignalP HMM score cut-off of $>0.9$ and NN cleavage site within 10 and 40 amino acids. Meanwhile, secreted proteins were predicted to contain RxLR motifs when RxLR position was present within 30 and 60 amino acids, RxLR position was higher than NN cleavage site, and signal peptide length was $\leq 30$.

$\mathrm{dN}$ and $\mathrm{dS}$ were calculated with TBtools (v0.66831). The background file was made from reference genome including location of each gene. The information files were made from predicted genome using homologous SNPs of each isolate, which contained the sequence of each gene from reference genome and the other isolates. We selected the corresponding function " $\mathrm{Ka} / \mathrm{Ks}$ calculation" of TBtools (v0.66831) and acquired the dN/dS data from each isolate as reported (Chen et al. 2020). Meanwhile, Figure 1D was made with R language from reported scripts (Birch et al. 2014). Patterns of gene positive selection is frequently observed in RxLR effector genes in Phytophthora. Here we used the same methods as reported before (Cooke et al. 2012; Raffaele et al. 2010a).

RNA sequencing and analysis. Total RNA was extracted from mycelium and infected potato leaves at 2 and 3 days after the inoculation of each isolate using Omega Total RNA Kit I according to the manufacturer's manual. RNA-seq service was provided by BGI. Analysis of gene expression was performed using HISAT2 for mapping, and HTSeq for counting reads on each gene. All statistical analyses were conducted in the R Environment (v3.6.0). Statistical analyses and plotting were performed in R server using the DESeq2 (v1.25.7), RcolorBrewer (v1.1-2), gplots (v3.0.1.1), amap (v0.817), ggplot2 (v3.2.0), and BiocParallel (v1.17.18) packages. A documented R-script and the data needed to reproduce figures and analyses were described in the Supplementary protocol and Supplementary Tables S11 and S12. The significantly regulated genes at 2 and 3 days postinoculation (dpi) among 06_3928A, HB1501, and HN1602 in Figure 2 were selected under the following conditions: $P$ value $<0.05$, expression fold change $>16$. Meanwhile, the specifically induced effectors of HB1501 and HN1602 were screened under the following conditions: sum read counts at 2 to 3 dpi of 06_3928A $\leq 2$ and sum read counts at 2 to 3 dpi of HB1501 and HN1602 $\geq 5$ of each gene. The gene ontology (GO) enrichment analysis was performed using the OmicShare tools, a free online platform for data analysis (https://www.omicshare. com/tools).

Effector transient expression and infection assay. Nicotiana benthamiana is a host for $P$. infestans and a model for functional studies in the family Solanaceae. As such, it has been extensively used to investigate host and pathogen gene functions in P. infestans-plant interactions (Bozkurt et al. 2012; Saunders et al. 2012). To investigate whether $P I T G_{-} 11350$ could enhance the growth of $P$. infestans during infection, we used an in planta transient assay (McLellan et al. 2013). In this assay, GFP-PITG_ 11350 was expressed in one half of $N$. benthamiana leaves via Agrobacterium tumefaciens mediated transient transformation (agroinfiltration) $24 \mathrm{~h}$ prior to inoculation with P. infestans $06_{-}$ 3928A zoospores; the second half of the leaves expressed the empty vector control (GFP alone). Four days postinoculation, the mean lesion size and mean necrosis size sporulating were recorded with method from the previous report (King et al. 2014).

Confocal microscopy and western blot assays. For these experiments, we take the same methods from a previous report (Gao et al. 2020). Confocal microscopy observation was performed on the $N$. benthamiana leaf patches 2 days postinfiltration by LSM 710 laser-scanning microscope with $\times 20 / 0.8$ or $\times 40 / 0.95$ objective lens (Carl Zeiss). The GFP and autofluorescence were scanned under excitation of 488- and 514-nm laser with emissions collected between 535 and $636 \mathrm{~nm}$. The conditions for microscopy data acquisition were consistent across samples. The images were processed by ZEISS ZEN Microscope Software and Adobe Photoshop at the same time.

For the western blot assays, proteins were expressed in $N$. benthamiana and extracted by the $\mathrm{PH}$-increased lysis buffer $(150 \mathrm{mM} \mathrm{NaCl}, 10 \mathrm{mM}$ Tris- $\mathrm{HCl} \mathrm{pH}=8.3,0.5 \mathrm{mM}$ ethylenediaminetetraacetic acid [EDTA], and $1 \%$ Triton X-100) with $1 \%$ protease inhibitor mixture (Sigma-Aldrich, P9599). Proteins were detected by anti-GFP (1:2,000, Abmart, P30010) antibody. All of the experiments were repeated three times with similar results.

\section{RESULTS}

Two newly emerged Chinese $P$. infestans isolates are virulent toward several resistant potato varieties. In order to monitor the diversity in Chinese $P$. infestans populations, we collected 384 isolates from the distinct geographic regions including Anhui, Fujian, Hebei, Hunan, Sichuan, and Yunnan Provinces from 2011 to 2016 ( $\mathrm{Li}$ et al. 2017a; Shu 2015). These isolates were grouped phenotypically (virulence type and mating type). We distinguished 11 groups and found two supervirulent isolates (overcoming R1 to R11 resistance) (Supplementary Table $\mathrm{S} 1$ ). This data suggests that filed isolates with similar virulence type and mating type occurred in different regions of China. For example, the isolate AH1409 from Anhui and FJ1411 from Fujian, which were both collected in the year 2014, belong to the same group. Most representative groups occur in at least $25 \%$ of each local field population in a particular year and region.

The frequency of Chinese supervirulent population in the field has a rapid change from nearly $1 \%$ (Yang et al. 2007) to nearly $10 \%$ (Supplementary Table S1) during these years. Both isolates (HB1501 and HN1602) displayed supervirulent phenotypes. They can overcome conventional R1 to R11 resistance but have different mating types. Interestingly, HB1501 was isolated in Hebei (North China) in the year 2015 and HN1602 was collected in Hunan (South 
China) in the year 2016. Therefore, we speculated that a deep study of these two representative isolates could provide insight into supervirulent isolates and find solutions for late blight disease management.
HB1501 and HN1602 have distinct genomic characteristics. To further investigate the virulence of the two isolates, we sequenced the genomes of HB1501 and HN1602 with Illumina Hi-Seq 2000 to $>50 \times$ coverage (Supplementary Table S2). Reads from HB1501 and
A

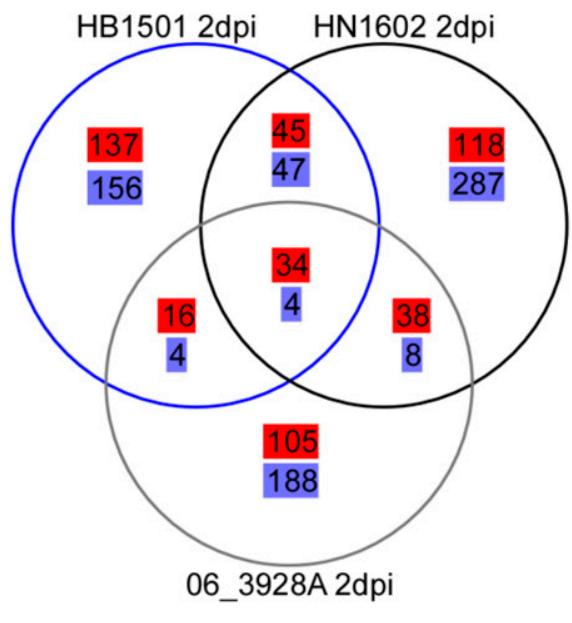

up-regulated down-regulated

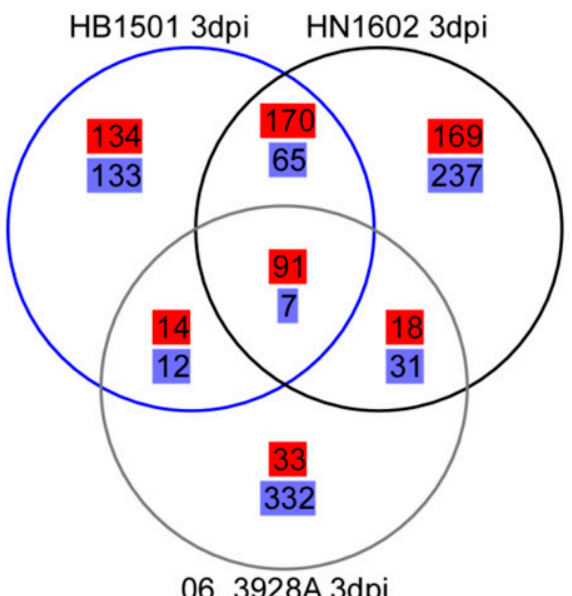

up-regulated

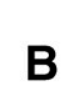

HB1501

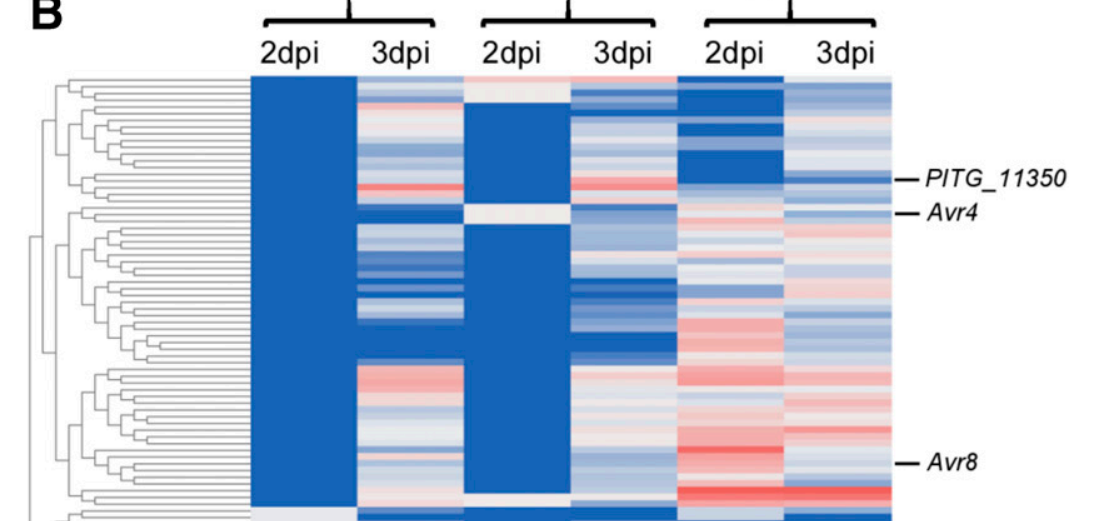

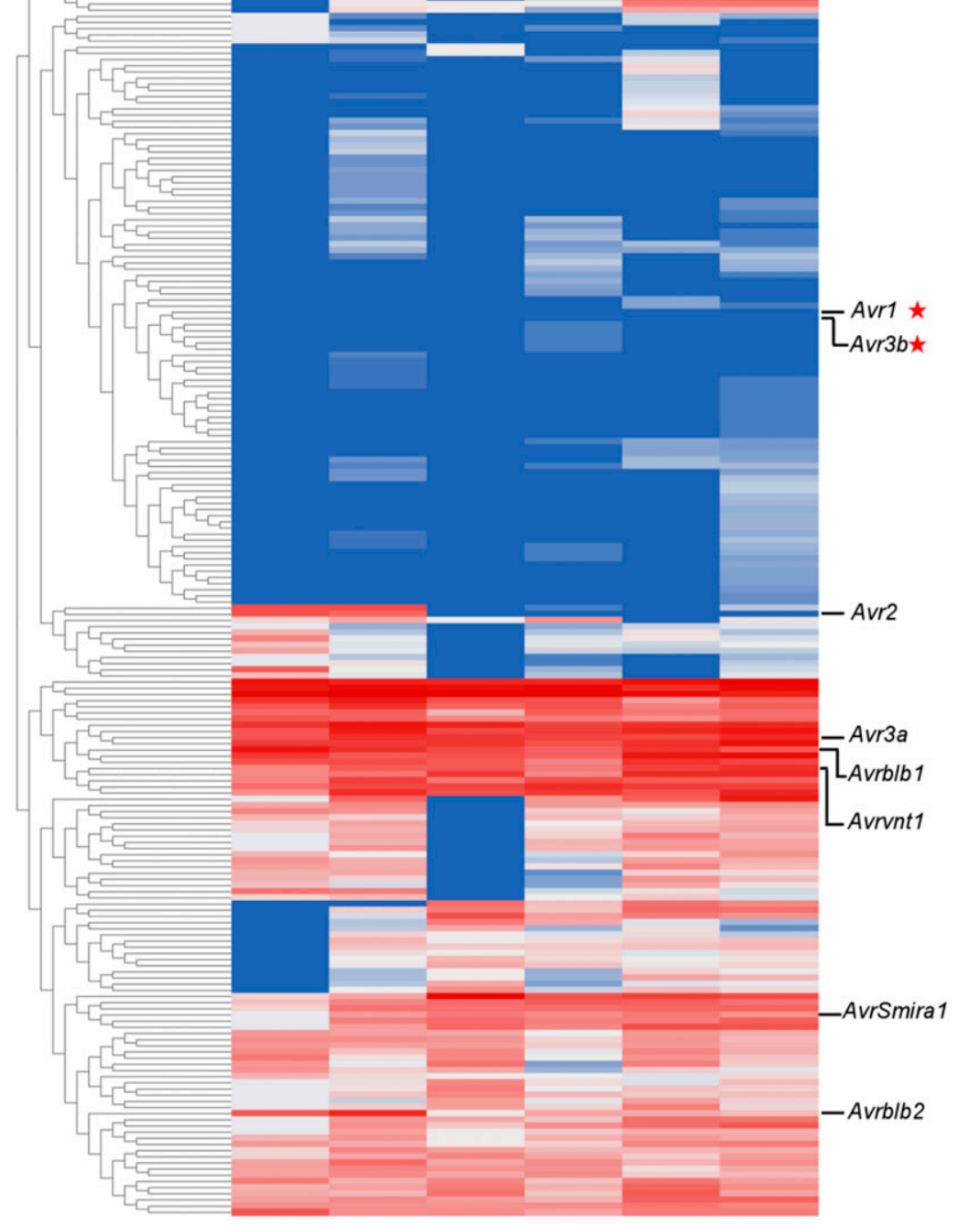

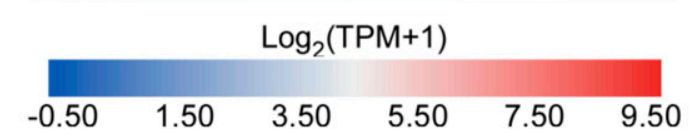

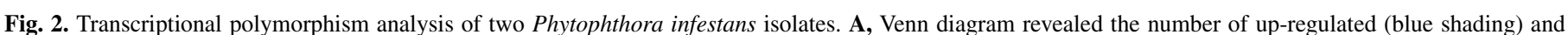

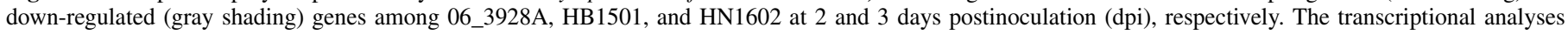

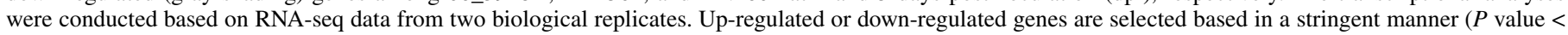

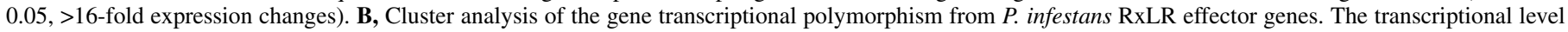

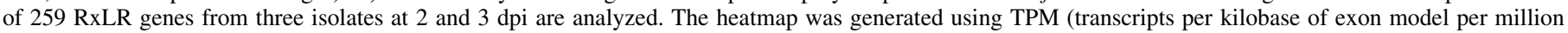

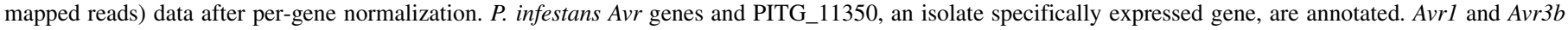
have no expression (red star) in these three isolates. 
HN1602 covered 98.52 and $98.74 \%$, respectively, of the reference genome T30-4 (Supplementary Table S2A). In order to examine the phylogenetic relationship of different isolates, we calculated the phylogeny based on genome sequencing data. Here, we extracted the sequence from scaffold 1.1, which is the largest scaffold in the reference genome for phylogeny construction (Martin et al. 2016). The phylogeny shows that HB1501 is close to DDR7062 (US-1 lineage), while HN1602 is clustered with 06_3928A (EU_13_A2 lineage), indicating that the two isolates have unrelated genetic backgrounds (Fig. 3).

$P$. infestans is diploid but different ploidy such as triploidy and tetraploidy are found in field isolates (Li et al. 2017b; Yoshida et al. 2013). Compared with diploid isolates, polyploid isolates have more alleles at the same genetic locus, thereby the studies of ploidy are important for the evolution of asexually reproducing organisms (Li et al. 2017b). Therefore, we investigated the genome-wide SNP frequency in both isolates. A normal SNP frequency distribution was observed in HB1501 and a bimodal distribution was found in HN1602, suggesting that HB1501 is a diploid whereas HN1602 is a triploid (Fig. 4A). The ploidy of HN1602 corresponds to the ploidy as reported for EU_13_ $\mathrm{A} 2$, which is in agreement with our phylogenetic analysis
(Fig. 3). This further confirmed that Chinese P. infestans populations from different regions have different genetic compositions.

Sequence polymorphisms within HB1501 and HN1602 genomes. To further characterize the two isolates genetically, we compared the genome sequences of HB1501 and HN1602 with 06_ 3928A (Cooke et al. 2012) and T30-4 reference genomes (Haas et al. 2009). The number of genes with copy number variations (CNVs) within HB1501, HN1602, and 06_3928A was 383, 304, and 235 , respectively. The number of gene deletions was 139,92 , and 43, respectively (Fig. 4B). To systematically analyze genetic polymorphisms in the genomes of HB1501 and HN1602, we identified SNPs of each isolate relative to the reference genome. In total, 234,988 SNPs were detected in the HB1501 genome, 206,972 SNPs were present in the noncoding sequence, 28,016 SNPs were found in 5,900 genes, and 50.7\% of the SNPs caused nonsynonymous mutations. In the HN1602 genome, 202,815 SNPs were detected, 180,287 SNPs in noncoding sequences while 22,528 SNPs were found in 5,582 genes, and $50.8 \%$ of the SNPs caused nonsynonymous mutations. Similarly, our analyses in 06_3928A captured 163,212 SNPs. In total, 142,115 SNPs were present in noncoding sequences, 21,097 SNPs were found on 5,549 genes, and

Tree scale: 0.1

\section{EU_13_A2 lineage \\ US-1 lineage \\ P. mirabilis \\ Chinese \\ South American \\ Mexico \\ Scotland \\ Netherlands \\ Hungary}

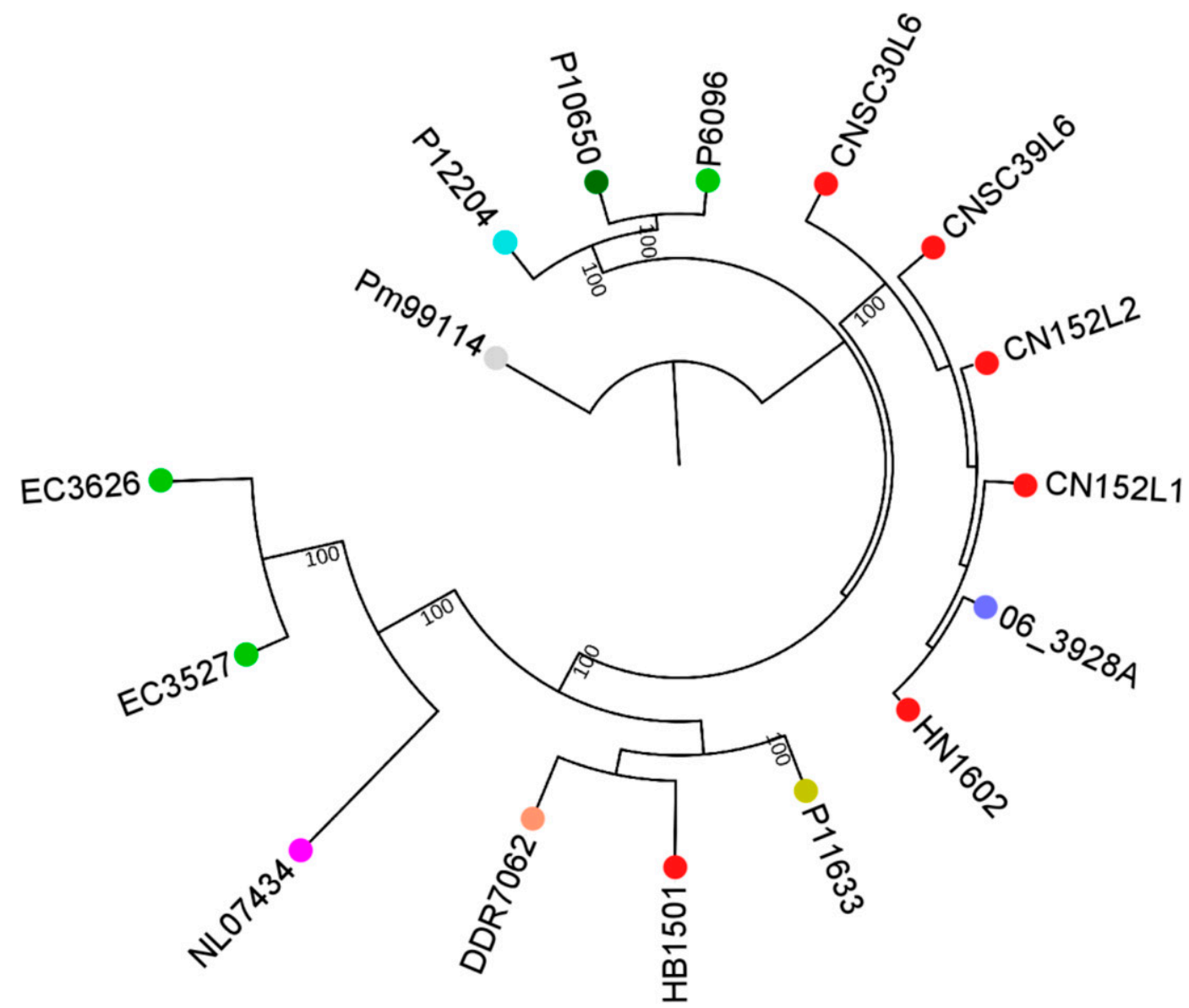

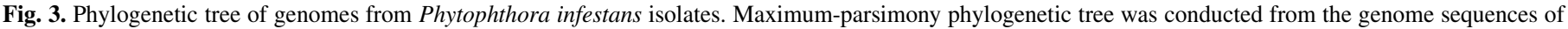

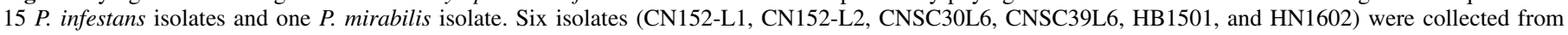

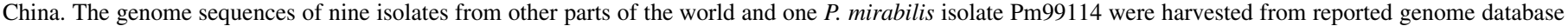
(Cooke et al. 2012; Li et al. 2017b; Yoshida et al. 2013). 
$50.3 \%$ of the SNPs caused nonsynonymous mutations (Fig. 4C, Supplementary Table S2B).

To compare the evolutionary selection pressure in coding sequences between the different isolates, we counted the nonsynonymous ( $\mathrm{dN}$ ) and synonymous (dS) substitution rates within each isolate (Fig. 4D and $\mathrm{E})$. The $\mathrm{dN}$ of $\mathrm{HB} 1501$ was 0.00089 and the $\mathrm{dS}$ was 0.0023 , while the $\mathrm{dN}$ of $\mathrm{HN} 1602$ was 0.00076 and the $\mathrm{dS}$ was 0.0019 . As a comparison, the $\mathrm{dN}$ of 06_3928A was 0.00067 and the dS was 0.0018 . The genome-wide $\mathrm{dN}$ and $\mathrm{dS}$ rates of the two Chinese isolates were higher than those of 06_3928A from the United Kingdom.
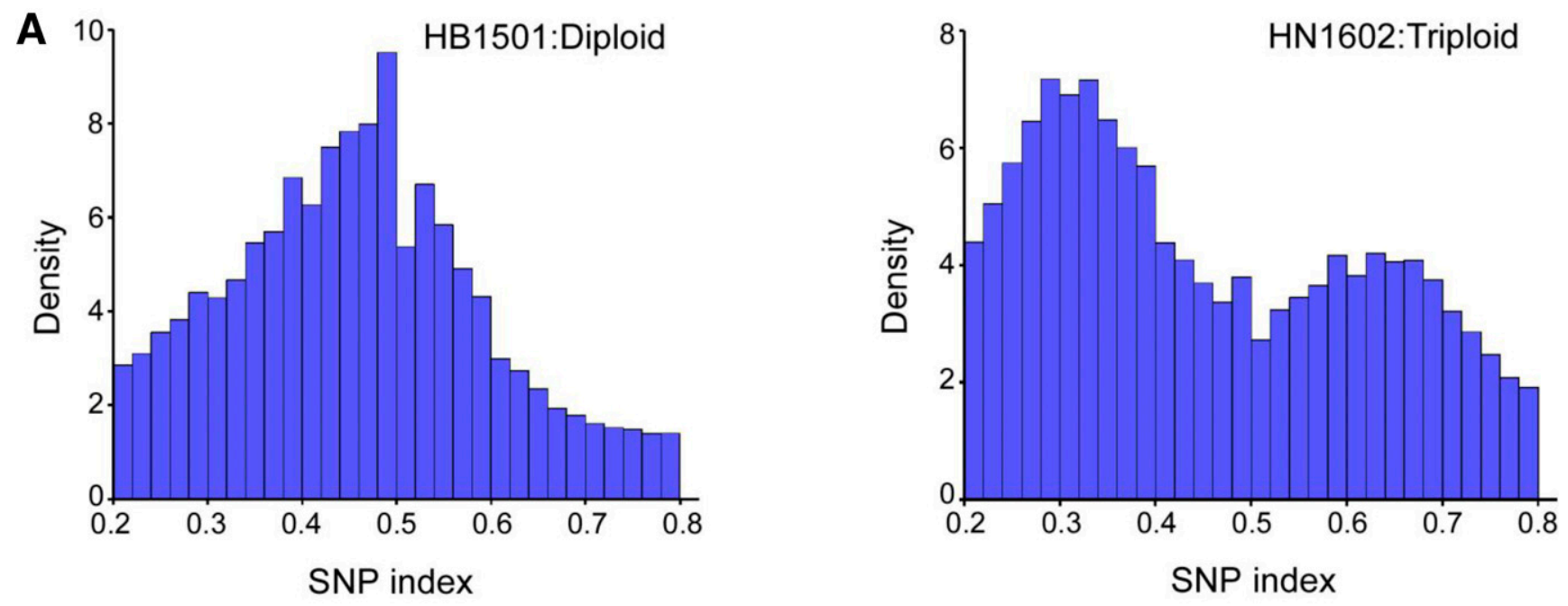

B
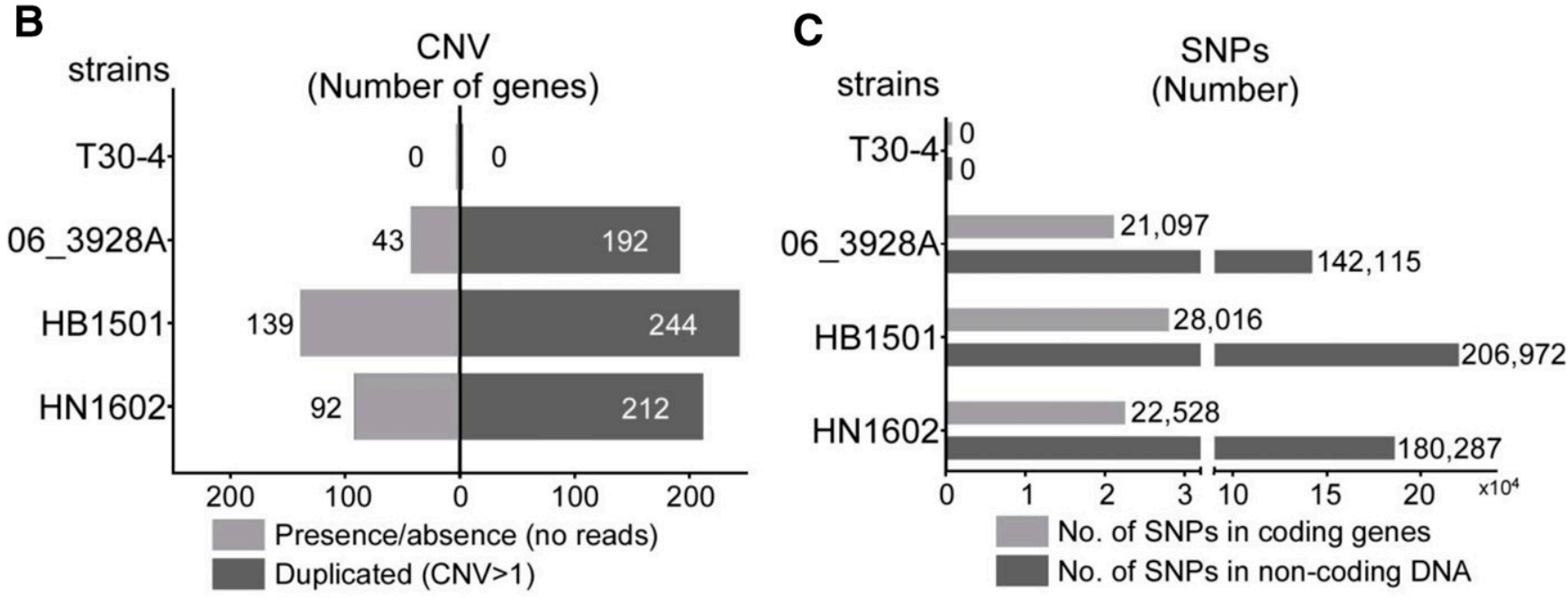

$\mathrm{dN}$ in T30-4 CDSs
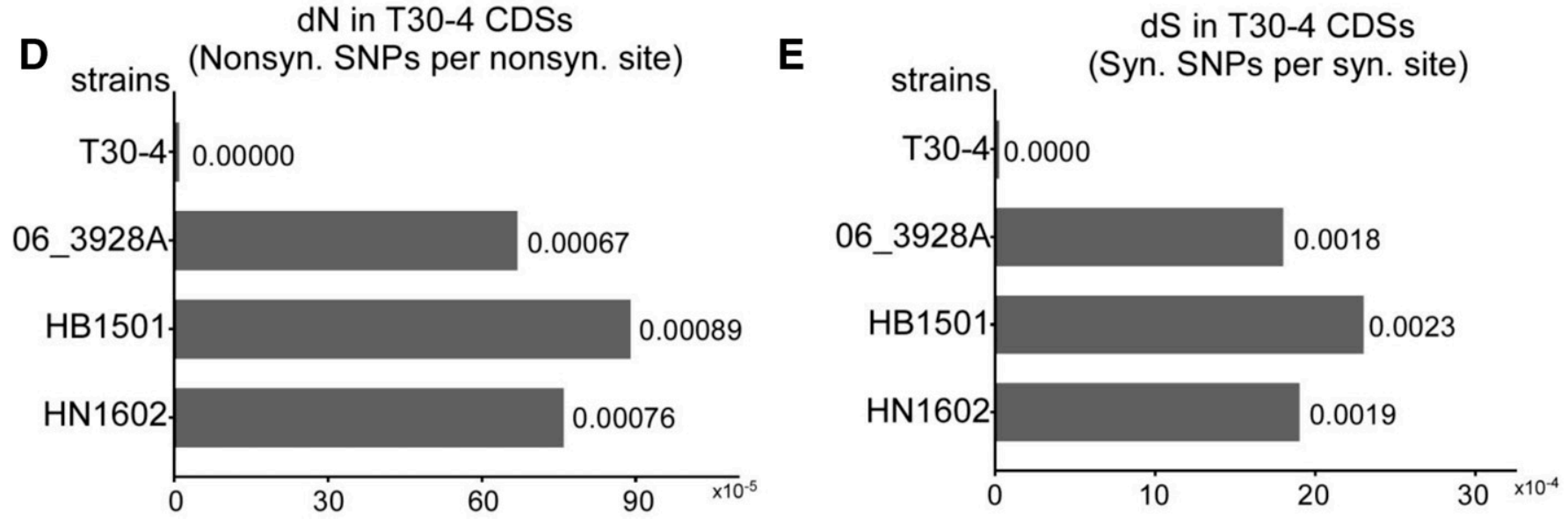

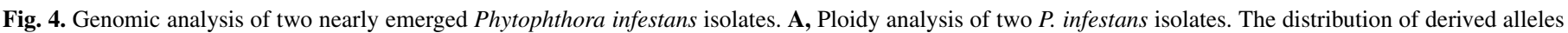

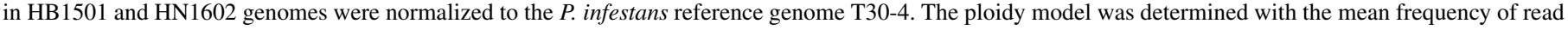

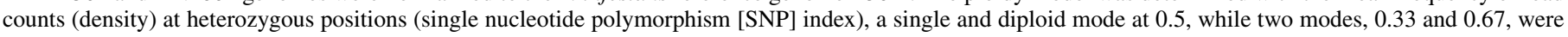

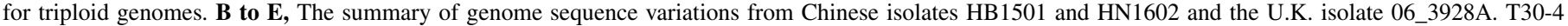
genome was included as control. CDS, coding sequence; CNV, copy number variation; and Syn. and Nonsyn., synonymous and nonsynonymous, respectively. 
We then studied the distribution of sequence polymorphisms among the known functional genes from different isolates. The proportion of CNVs among genes of the conserved core orthologs and intercellular proteins from the two isolates was relatively low $(<1 \%)$ and not significantly different from that of 06_3928A (Fig. 1A). However, CNV drastically rose to 2 to $8 \%$ in genes encoding secreted proteins, RxLR and CRN effectors, as well as proteins involved in epigenetic regulation. These proteins are expected to contribute to the aggressiveness and virulence phenotype. In addition, both RxLR and CRN effector encoding genes showed higher percentages of nonsynonymous SNPs (Fig. 1B). From the analysis of genes under positive selection $(\mathrm{dN} / \mathrm{dS}>1)$, we found positively selected genes are mostly enriched in RxLR effector gene in all three examined isolates (Fig. 1C). Besides, we found 60 genes from HB1501 and 56 genes from HN1602 were under positive selection, and these genes mostly reside in the GSR (Fig. 1D). These results suggested that the effector genes, in particular RxLR effector genes, are positively selected in the two isolates.

To predict potential new RxLR effectors in the genomes of both genotypes, we assembled the reads that did not map to the T30-4 reference genome and obtained 16.0 and $16.5 \mathrm{Mb}$ of contigs from HB1501 and HN1602, respectively. We finally mined putative RxLR effector genes out of those additional sequences as five genes from HB1501 and four genes from HN1602 (Supplementary Table $\mathrm{S} 3$ ). A reciprocal BLAST confirmed that these RxLR effector genes are indeed absent from the T30-4 reference genome. Among these genes, Pex14182 and Pex50259 have been previously identified in U.K. isolate 06_3928A. While Pex002 and Pex003 are present in both Chinese isolates but absent from 06_3928A and the reference genome. Pex001 gene is only present in HB1501.

Transcriptome analyses of HB1501 and HN1602. To further examine the global gene expression level during host infection, we profiled the transcriptome landscape from in vitro growth and the potato leaves 2 and 3 days after inoculation. RNAseq was performed by Illumina sequencing and yielded satisfying sequencing output (Supplementary Table S4). To assess the consistency between biological replicates and the degree of variation among different samples, we performed principal components analysis (PCA) analysis. The PCA data suggested that the three isolates had a higher transcriptional correlation during in planta growth than in the in vitro growth (Supplementary Fig. S1).

To further investigate the transcriptional differences during infection, we counted the up-regulated genes of HB1501, HN1602, and 06_3928A at different infection time points. At 2 dpi, 232, 235, and 193 genes were up-regulated significantly, while 211,346 , and 204 genes were down-regulated significantly in HB1501, HN1602, and 06_3928A, respectively. At 3 dpi, 409, 448, and 156 genes were up-regulated significantly, while 217, 340, and 382 genes were down-regulated in HB1501, HN1602, and 06_3928A, respectively (Fig. 2A). Furthermore, we observed that 34 genes were significantly up-regulated, and four genes were down-regulated in all three isolates at 2 dpi. We also noticed 91 genes were upregulated and seven genes were down-regulated significantly in all three isolates at $3 \mathrm{dpi}$. So, the three isolates share a small number of coregulated genes and most up-regulated and down-regulated genes are only observed in single isolates. Meanwhile, we found 257 RxLR effector genes were expressed during the infection stage within different isolates (Fig. 2B). However, we counted 55 coregulated RxLR effector genes at 2 dpi and 150 coregulated RxLR effector genes at 3 dpi (Fig. 2B, Supplementary Table S11). This observation suggested that the $P$. infestans isolates under study display significant gene expression plasticity.

We further examined the genes that are specifically induced in the two Chinese isolates. Among 817 genes that were specifically induced in either HB1501 or HN1602 during infection (Supplementary Table S9), 113 genes had clear annotation and were potentially involved in virulence (Supplementary Fig. S2A, Supplementary Table S12). The encoding proteins included 46 intercellular proteins, $44 \mathrm{CRN}$ effectors, 16 RxLR effectors, and 7 enzymes that were potentially involved in epigenetic regulation. Those 46 intercellular proteins include 27 glycoside hydrolase (GHs), 10 polysaccharide lyases (PLs), 5 serine proteases (SPs), and 4 other enzymes (Supplementary Fig. S2B). In summary, these results showed that the transcriptome data had high reproducibility and reflected the basic biological characteristics of different $P$. infestans isolates in their interaction with host plants.

Biological function of specifically induced RxLR effector gene $P I T G \_11350$ during infection is correlated with virulence. To further investigate the impact of specifically induced effector genes during potato infection of the two Chinese isolates, we focused on genes that were significantly up-regulated during infection. First, we observed the transcript abundance of specifically induced RxLR effectors. PITG_11350, which is a member of the reported PexRD2 family (Haas et al. 2009), showed the strongest up-regulation among specifically induced RxLR genes at 3 dpi (Fig. 5A). As reported before, the P. infestans RXLR effector PexRD2 interacts with the kinase domain of MAPKKK, a positive regulator of cell death associated with plant immunity (King et al. 2014). To assign potential biological functions of $P I T G_{-} 11350$ regulation in the Chinese isolates, we performed a transcomplementation experiment. $N$. benthamiana was first agroinfiltrated to transiently express GFP-PITG_11350 and afterward challenged with 06_3928A that did not induce PITG_11350 expression itself. After 4 days, enhanced lesion growth and necrosis were observed when compared with the GFP only as control (Fig. 5B and C). This result demonstrated that specifically induced RxLR effector of two Chinese isolates could promote effector-triggered susceptibility and contribute to virulence.

Polymorphisms of $A V R$ effector genes are correlated with virulence phenotype of Chinese field isolates. Oomycete pathogens secrete host-translocated RxLR effectors that include avirulence (AVR) proteins, which are perceived by $R$ proteins from wild Solanum species. To explore which variations of $A v r$ genes might be associated with pathogen adaptation among HB1501 and HN1602, we examined absence of gene, gene mutations, and silencing of known Avr genes (Fig. 6A). The results revealed that Avrl (PITG_16663) was absent in both HB1501 and HN1602. Avr3b (PITG_18215) was absent in HN1602 but present in HB1501. Therefore, we performed RT-PCR experiments (Fig. $6 \mathrm{~B}$ ), which revealed gene silencing as a recognition evasion mechanism that had not been described before for $A v r 3 b$ ( $\mathrm{Na}$ et al. 2014; Pais et al. 2018). To characterize virulence phenotype further, we compared sequence polymorphisms and expression profiles of known $A v r$ genes with the reference genome and reported virulent mutations. The results showed that $A V R 3 a\left(P I T G \_14371\right)$ and $A V R 4$ $\left(P I T G \_07387\right)$ had virulence causing mutations that were reported before (Armstrong et al. 2005; van Poppel et al. 2008). Importantly, no mutations were found in Avrblb1, Avrblb2, Avr8, and Avrvnt1, which were up-regulated in HB1501 and HN1602 during infection of susceptible potato. In summary, we found HB1501 and HN1602 evade recognition of $\mathrm{R} 1, \mathrm{R} 3 \mathrm{a}, \mathrm{R} 3 \mathrm{~b}$, and $\mathrm{R} 4$, but they still express the whole sequence of Avr8, Avrvnt1, Avrblb1, and Avrblb2, which is greatly important for the deployment of the corresponding $R$ genes in potato varieties for agriculture.

Pinpointing valuable potato resistance to manage potential HB1501 and HN1602 epidemics. To confirm the (a) virulence prediction from the RNA-seq experiments, we assessed the virulence of HB1501 and HN1602 on isogenic resistant potato plants (Zhu et al. 2014). Potato plants with $R 3 a$ and/or $R 3 b$ were susceptible to HB1501 and HN1602 inoculation. However, potato plants with Rpi-blb1, Rpi-blb2, R8, and Rpi-vnt1.1 were resistant to HB1501 and HN1602 (Fig. 6C). Furthermore, HB1501 and HN1602 have different virulence on resistant plants that recognize $A v r 2$. The results revealed that HB1501 can be recognized by Rpi-mcql and Rpi-blb3, while HN1602, which lacks $A v r 2$ sequences, can overcome resistant potato containing Rpi-mcql 
but not Rpi-blb3 in a similar way as observed previously (AguileraGalvez et al. 2018).

Our initial analysis showed that the two Chinese isolates successfully escaped recognition by $\mathrm{R} 1$ to $\mathrm{R} 11$ differential set plants through gene deletion, sequence polymorphisms, or gene silencing. However, these newly emerged isolates still secrete other conserved RxLR effector proteins that are up-regulated during the infection phase, which could be recognized by the novel, so far unidentified, $\mathrm{R}$ proteins.

\section{DISCUSSION}

Polyploidization enhances allelic diversity in the absence of frequent sexual reproduction for adapting to the environment (Yoshida et al. 2013). Our genome-wide analysis data demonstrated that HB1501 is diploid while HN1602 is triploid, and HB1501 is closer to the progeny from US-1 lineage, whereas HN1602 clustered with 06_3928A, a isolate from 13_A2 lineages. Similarly, it was reported that one of the representative clonal lineages from China was genetically similar to 06_3928A (Li et al. 2012). However, HN1602 shows differences from 06_3928A as we presented genes with clear sequence and transcriptional polymorphisms. It would be interesting to further study the evolution of HN1602-like population. Hybridization is important for adaptation to new hosts in several fungal and oomycete pathogens (Goss et al. 2011), and $P$. infestans can sexually reproduce resulting in overwintering oospores, further increasing the variability and severity of the pathogen (McDonald and Linde, 2002). It is also worth noting that the mating type of triploid HN1602 is A1A2, suggesting that HN1602 could proliferate in the field through the asexual sporangia, but also conduct sexual reproduction through the production of oospores.

Our results suggest that the Avr effectors of these newly emerged $P$. infestans isolates take different strategies to escape the recognition from corresponding $\mathrm{R}$ proteins (Supplementary Table S5). These strategies include the deletion of $A v r l$ and $A v r 3 b$, nucleotide polymorphisms in $A v r 3 a$ and $A v r 4$, and transcriptional silencing of $A v r 3 b$ (Fig. 6A). It is known that both $A v r 3 a^{E M}$ allele and $A v r 4$ allele with the frameshift mutation evade the recognition of corresponding R proteins (Armstrong et al. 2005; van Poppel et al. 2008). Consistent with those reports, we found both isolates gain virulence on $R 3 a$ transgenic potato. Previous reports showed that presence/absence and SNP polymorphisms of $A v r 2$ evade R2 recognition (Gilroy et al. 2011; Turnbull et al. 2019). Here, we found that $A v r 2$ avirulent allele $A v r 2^{31 K}$ is present in isolate HB1501 but none of the $A v r 2$ alleles can be found in HN1602. This explains why Rpi-mcq1, an R protein recognizing Avr2, provides resistance to HB1501 but not HN1602. However, transgenic potato expressing Rpi-blb3, another $\mathrm{R}$ protein from a distinct NLR family that recognizes Avr2, confers resistance to both isolates (Fig. 6C). Given the presence of $A v r 2$ homologous genes in both isolates, we reason that Rpi-blb3 may recognize $A v r 2$ homologs and thereby activate potato resistance. Our data are in agreement with a previous observation that Rpi-blb3 confers resistance to several isolates such as NL12003 and NL09096, which Rpi-mcq1 fails to recognize (Aguilera-Galvez et al. 2018).

Interestingly, both isolates show clear virulence on $R 3 b$ potato. Given that the DNA sequences in $A v r 3 b$ loci are identical as $444 \mathrm{bp} /$ 447 bp (99.3\%) between HB1501 and T30-4, whereas the gene expression differs, we speculated that $A v r 3 b$ silencing maybe subjected to epigenetic variation in isolate HB1501. Emerging evidence suggests that gene silencing is a flexible strategy deployed by the pathogen to breakdown host resistance. The previous study showed that Avrvnt1 gene silencing causes evasion of Rpi-vnt1.1 mediated recognition in a $P$. infestans isolate P13626 from EC-1 clonal lineage (Pais et al. 2018). In another report, $A v r 3 b$ gene silencing in an isolate was also documented (Rietman 2011). However, the mechanism underlying gene silencing remains unclear. Avr gene silencing mechanisms were investigated in $P$. sojae and showed where PsAvr $3 a$ silencing is associated with abundance of 24 to $25 \mathrm{nt}$ sRNAs and PsAvrlb silencing is mediated by histone H3K27me3 modification (Qutob et al. 2013; Wang et al. 2019). Chen and colleagues reported that adenine N6-methylation $(6 \mathrm{~mA})$, a putative epigenetic mark, is associated with the silenced genes in both $P$. infestans and $P$. sojae (Chen et al. 2018). Previous mechanistic studies have revealed that INF1 gene silencing in $P$. infestans can be released by chemical inhibitors of DNA methylation and histone deacetylation (van West et al. 2008). Whether $A v r 3 b$ gene silencing is mediated by epigenetic variation remains for future investigation.

Our study highlighted that the genes under sequence and transcriptional polymorphisms in HB1501 and HN1602 are mainly enriched in the RxLR effector gene category. This is not surprising as RxLR effector genes playing roles in interaction with host plants demonstrate accelerated gene evolutionary signatures. We also certify that there is no bias of gene length with positive selection in RXLR effectors (Supplementary Table S15). The effector gene PITG_11350 we studied here belongs to $P$. infestans PexRD2 gene family and PexRD2 (PITG_11383) effectors manipulate plant MAPK signaling pathways (Haas et al. 2009; King et al. 2014). Whether PITG_11350 blocks potato MAPK signaling or it can be recognized by some unidentified plant $R$ genes remains unclear.
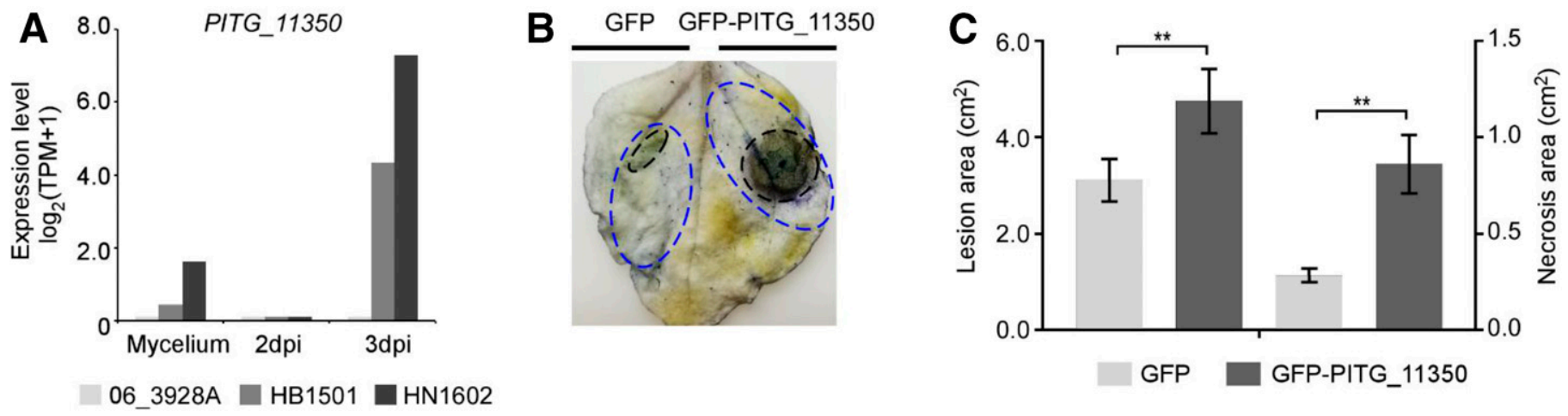

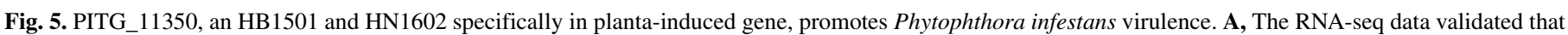

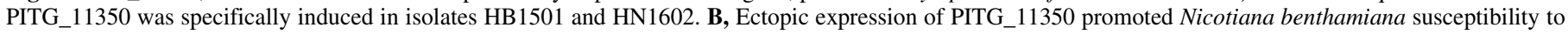

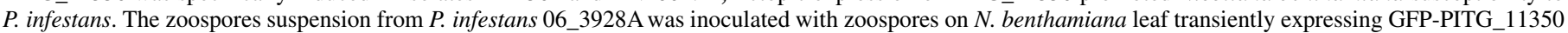

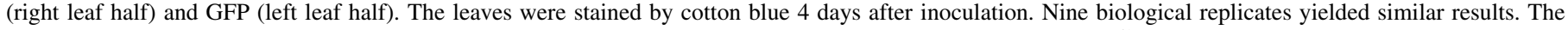

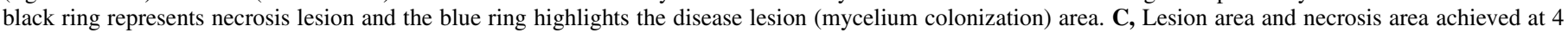

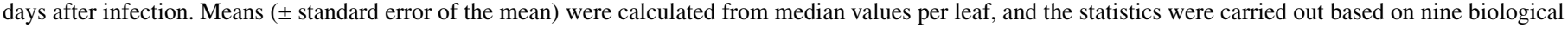
replicates. ${ }^{* *}, P$ value $<0.01$. 
Furthermore, our GO enrichment analyses revealed that predicted polysaccharide lyase (PL) family genes are significantly enriched in the specifically induced genes in both isolates. PL proteins are known to contribute to the pathogenicity in oomycete, fungal, and bacterial (Fu et al. 2015; Li et al. 2019). Therefore, we reason that specifically induced PL proteins may accelerate pathogen ability to degrade plant cell wall and consequently contribute to the aggressiveness of field isolates. However, this hypothesis needs to be further studied in the future. Our results suggest that the newly emerged $P$. infestans isolates activate distinct gene expression landscapes of effector repertoires to adapt on potato plants in the field.

Matching the filed isolates virulence types and the dominance of $R$ genes that potato varieties deployed in certain regions would provide more insight into the selection of both isolates and varieties at the molecular level. As reported before, the composition of $R$ genes in 29 potato varieties was analyzed by Agrobacteriummediated transient expression technique with eight $A v r$ genes as tools, but the results are somewhat conflicting and confusing (Guan et al. 2015; Ma et al. 2020). Nevertheless, phenotyping and genotyping the emerging field isolates could provide clues for rational disease control by deploying cognate $R$ gene sets. Besides $R p i$-blb3, our data also showed that potato $R$ genes $R 8, R p i$-vnt1.1, $R p i-b l b 2$, and $R B$ confer resistance to the two isolates, suggesting the potato varieties carrying these $R$ genes could be used to fight against newly emerged filed isolates. The recognition by $R 8$ was unexpected as both isolates were characterized as supervirulent because of their behavior on the R1 to R11 differential set plants, that includes $R 8$. We speculate that this was caused by distinct genetic compositions from different plants. It has been observed before that $R 8$ mediated resistance depends on other genes in the genetic background and assay type (Jo et al. 2011; Zhu et al. 2014). Recent studies revealed that some Avrblbl (IPI-O) family members are able to evade $\mathrm{RB}$ detection and even IPI-O4 blocks $\mathrm{RB}$ recognition, leading to the inactivation of $\mathrm{RB}$-mediated programmed cell death (Champouret et al. 2009; Chen et al. 2012). Both HB1501 and HN1602 express IPI-O1 and IPI-O2 homologs, but IPI-O4 was not detected in the genome of HB1501 or HN1602 (Supplementary Fig. S5). In summary, these data indicated that $R B$, $R 8$, and Rpi-vntl.1 are valuable $R$ genes for managing newly
A

HB1501

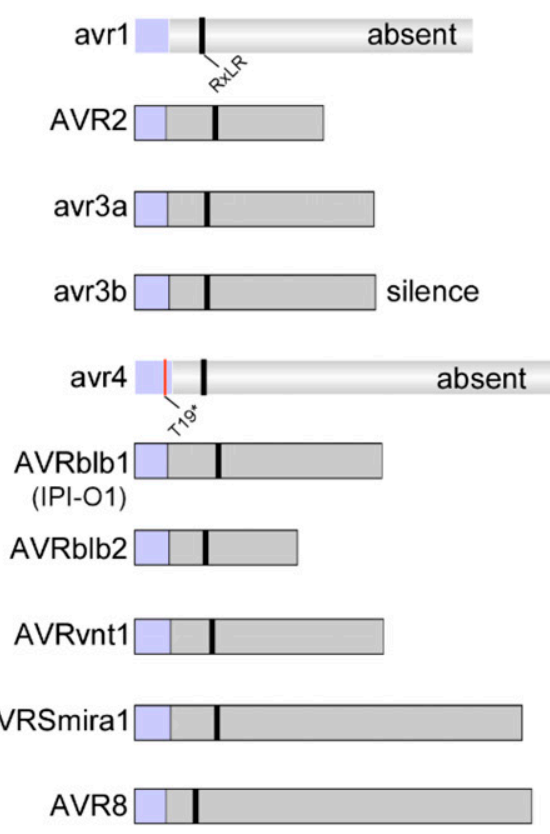

Expression patterns

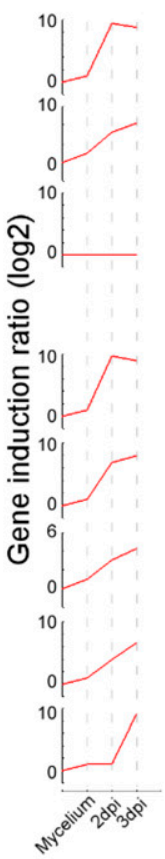

HN1602

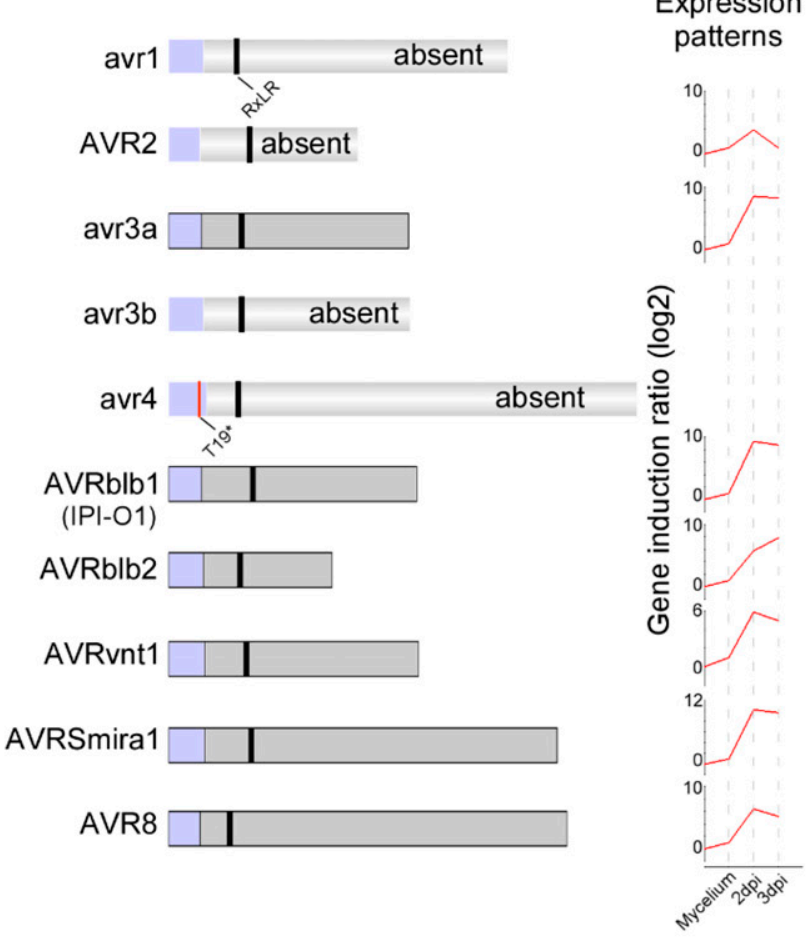

B

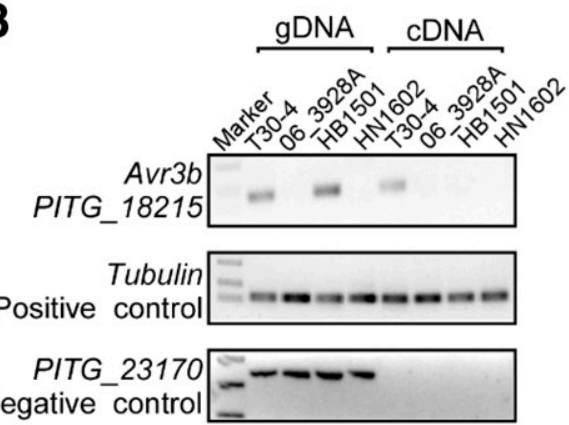

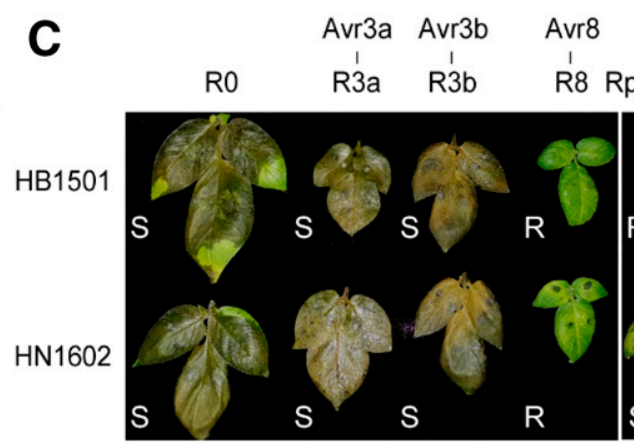

Avr8 Avr2

Avrvnt1 Avrblb2 Avrblb1 Rpi-vnt1.1 Rpi-blb2 Rpi-blb1

Fig. 6. The polymorphisms of $A v r$ genes in two isolates and the infection assay on isogenic potato lines carrying $R$ genes reveal the valuable potato resistance in late blight management. A, The summary of sequence polymorphisms and expression patterns of Avr genes in two Chinese isolates HB1501 and HN1602. B, RT-PCR experiments validated $A v r 3 b$ silencing in isolate HB1501. Tubulin was used as a positive control, while PITG_23170 was taken as a negative control. Avr3b is absent in the genome of 06_3928A and HN1602. C, Several isogenic potato lines carrying different $R$ genes confer resistance to HB1501 and HN1602. R indicates potato leaves were resistant to corresponding Phytophthora infestans, and $\mathrm{S}$ indicates potato leaves were susceptible to corresponding $P$. infestans. 
emerged isolates in the field. However, individually deploying these genes is risky as they may lose value rapidly, and we should deploy them carefully using rational strategies such as pyramiding and rotations.

\section{ACKNOWLEDGMENTS}

We thank Qian Zhou from Sanwen Huang's lab (Chinese Academy of Agricultural Sciences) for sharing genome sequence data; Xiong Zhang and Haidong Shu (Nanjing Agricultural University) for bioinformatics support; and the Bioinformatics Center of Nanjing Agricultural University for bioinformatics technical support.

\section{LITERATURE CITED}

Aguilera-Galvez, C., Champouret, N., Rietman, H., Lin, X., Wouters, D., Chu, Z., Jones, J. D. G., Vossen, J. H., Visser, R. G. F., Wolters, P. J., and Vleeshouwers, V. G. A. A. 2018. Two different $R$ gene loci co-evolved with Avr 2 of Phytophthora infestans and confer distinct resistance specificities in potato. Stud. Mycol. 89:105-115.

Armstrong, M. R., Whisson, S. C., Pritchard, L., Bos, J. I. B., Venter, E., Avrova, A. O., Rehmany, A. P., Bohme, U., Brooks, K., Cherevach, I., et al. 2005. An ancestral oomycete locus contains late blight avirulence gene Avr3a, encoding a protein that is recognized in the host cytoplasm. Proc. Natl. Acad. Sci. USA 102:7766-7771.

Birch, P., Jones, J. T., and Bos, J. I. B. 2014. Plant-Pathogen Interactions: Methods and Protocols. Humana Press, New York.

Birch, P. R. J., Armstrong, M., Bos, J., Boevink, P., Gilroy, E. M., Taylor, R. M., Wawra, S., Pritchard, L., Conti, L., Ewan, R., et al. 2009. Towards understanding the virulence functions of RXLR effectors of the oomycete plant pathogen Phytophthora infestans. J. Exp. Bot. 60:1133-1140.

Birch, P. R. J., Bryan, G., Fenton, B., Gilroy, E. M., Hein, I., Jones, J. T., Prashar, A., Taylor, M. A., Torrance, L., and Toth, I. K. 2012. Crops that feed the world 8: Potato: are the trends of increased global production sustainable? Food Secur. 4:477-508.

Bozkurt, T. O., Schornack, S., Banfield, M. J., and Kamoun, S. 2012. Oomycetes, effectors, and all that jazz. Curr. Opin. Plant Biol. 15:483-492.

Bradshaw, J. E., Pande, B., Bryan, G. J., Hackett, C. A., McLean, K., Stewart, H. E., and Waugh, R. 2004. Interval mapping of quantitative trait loci for resistance to late blight [Phytophthora infestans (Mont.) de Bary], height and maturity in a tetraploid population of potato (Solanum tuberosum subsp. tuberosum). Genetics 168:983-995.

Champouret, N., Bouwmeester, K., Rietman, H., van der Lee, T., Maliepaard, C., Heupink, A., van de Vondervoort, P. J. I., Jacobsen, E., Visser, R. G. F., van der Vossen, E. A. G., Govers, F., and Vleeshouwers, G. A. A. 2009. Phytophthora infestans isolates lacking class I ipiO variants are virulent on Rpi-blb1 potato. Mol. Plant-Microbe Interact. 22:1535-1545.

Chen, C., Chen, H., Zhang, Y., Thomas, H. R., Frank, M. H., He, Y., and Xia, R. 2020. TBtools: An integrative toolkit developed for interactive analyses of big biological data. Mol. Plant 13:1194-1202.

Chen, H., Shu, H., Wang, L., Zhang, F., Li, X., Ochola, S. O., Mao, F., Ma, H., Ye, W., Gu, T., et al. 2018. Phytophthora methylomes are modulated by $6 \mathrm{~mA}$ methyltransferases and associated with adaptive genome regions. Genome Biol. 19:181.

Chen, Y., Liu, Z., and Halterman, D. A. 2012. Molecular determinants of resistance activation and suppression by Phytophthora infestans effector IPI-O. PLoS Pathog 8:e1002595.

Chisholm, S. T., Coaker, G., Day, B., and Staskawicz, B. J. 2006. Host-microbe interactions: Shaping the evolution of the plant immune response. Cell 124:803-814.

Cooke, D. E., Cano, L. M., Raffaele, S., Bain, R. A., Cooke, L. R., Etherington, G. J., Deahl, K. L., Farrer, R. A., Gilroy, E. M., Goss, E. M., et al. 2012. Genome analyses of an aggressive and invasive lineage of the Irish potato famine pathogen. PLoS Pathog 8:e1002940.

Dong, S., Raffaele, S., and Kamoun, S. 2015. The two-speed genomes of filamentous pathogens: Waltz with plants. Curr. Opin. Genet. Dev. 35: 57-65.

Dou, D. L., Kale, S. D., Wang, X., Jiang, R. H. Y., Bruce, N. A., Arredondo, F. D., Zhang, X. M., and Tyler, B. M. 2008. RXLR-mediated entry of Phytophthora sojae effector Avrlb into soybean cells does not require pathogen-encoded machinery. Plant Cell 20:1930-1947.

Fu, L., Zhu, C. Y., Ding, X. M., Yang, X. Y., Morris, P. F., Tyler, B. M., and Zhang, X. G. 2015. Characterization of cell-death-inducing members of the pectate lyase gene family in Phytophthora capsici and their contributions to infection of pepper. Mol. Plant-Microbe Interact. 28:766-775.

Gao, C., Xu, H., Huang, J., Sun, B., Zhang, F., Savage, Z., Duggan, C., Yan, T., Wu, C. H., Wang, Y., Vleeshouwers, V. G. A. A., Kamoun, S., Bozkurt,
T. O., and Dong, S. 2020. Pathogen manipulation of chloroplast function triggers a light-dependent immune recognition. Proc. Natl. Acad. Sci. USA. 117:9613-9620.

Gijzen, M., Ishmael, C., and Shrestha, S. D. 2014. Epigenetic control of effectors in plant pathogens. Front. Plant Sci. 5:638.

Gilroy, E. M., Breen, S., Whisson, S. C., Squires, J., Hein, I., Kaczmarek, M., Turnbull, D., Boevink, P. C., Lokossou, A., Cano, L. M., et al. 2011. Presence/absence, differential expression and sequence polymorphisms between PiAVR2 and PiAVR2-like in Phytophthora infestans determine virulence on $R 2$ plants. New Phytol. 191:763-776.

Goss, E. M., Cardenas, M. E., Myers, K., Forbes, G. A., Fry, W. E., Restrepo, S., and Grunwald, N. J. 2011. The plant pathogen Phytophthora andina emerged via hybridization of an unknown Phytophthora species and the Irish potato famine pathogen, $P$. infestans. PLoS One 6:e24543.

Guan, Y., Zhang, Y., Cheng, Y., and Shi, L. 2015. Distribution of late blight resistance genes in different potato cultivars. Highlights of Science Paper Online. 8:2251-2257.

Haas, B. J., Kamoun, S., Zody, M. C., Jiang, R. H. Y., Handsaker, R. E., Cano, L. M., Grabherr, M., Kodira, C. D., Raffaele, S., Torto-Alalibo, T., et al. 2009. Genome sequence and analysis of the Irish potato famine pathogen Phytophthora infestans. Nature 461:393-398.

Harutyunyan, S. R., Zhao, Z., Hartog, T., Bouwmeester, K., Minnaard, A. J., Feringa, B. L., and Govers, F. 2008. Biologically active Phytophthora mating hormone prepared by catalytic asymmetric total synthesis. Proc. Natl. Acad. Sci. USA 105:8507-8512.

Haverkort, A. J., Boonekamp, P. M., Hutten, R., Jacobsen, E., Lotz, L. A. P., Kessel, G. J. T., Vossen, J. H., and Visser, R. G. F. 2016. Durable late blight resistance in potato through dynamic varieties obtained by cisgenesis: Scientific and societal advances in the DuRPh project. Potato Res. 59: 35-66.

Jo, K. R., Arens, M., Kim, T. Y., Jongsma, M. A., Visser, R. G. F., Jacobsen, E., and Vossen, J. H. 2011. Mapping of the S. demissum late blight resistance gene $R 8$ to a new locus on chromosome IX. Theor. Appl. Genet. 123:1331-1340.

Jones, J. D., Vance, R. E., and Dangl, J. L. 2016. Intracellular innate immune surveillance devices in plants and animals. Science 354:aaf6395.

Jones, J. D. G., and Dangl, J. L. 2006. The plant immune system. Nature 444: 323-329.

Judelson, H. S. 1997. Expression and inheritance of sexual preference and selfing potential in Phytophthora infestans. Fungal Genet. Biol. 21: 188-197.

King, S. R. F., McLellan, H., Boevink, P. C., Armstrong, M. R., Bukharova, T., Sukarta, O., Win, J., Kamoun, S., Birch, P. R. J., and Banfield, M. J. 2014. Phytophthora infestans RXLR effector PexRD2 interacts with host MAPKKK epsilon to suppress plant immune signaling. Plant Cell 26: 1345-1359.

Li, L., Yan, S., Yuanchao, W., and Xiaobo, Z. 2017a. Biological characteristics of self-fertile Phytophthora infestans from Fujian and Hunan provinces. J. Nanjing Agric. Univ. 40:444-449.

Li, N., Lin, B., Wang, H., Li, X., Yang, F., Ding, X., Yan, J., and Chu, Z. 2019. Natural variation in ZmFBL41 confers banded leaf and sheath blight resistance in maize. Nat. Genet. 51:1540-1548.

Li, Y., Shen, H., Zhou, Q., Qian, K., van der Lee, T., and Huang, S. W. 2017b. Changing ploidy as a strategy: The Irish potato famine pathogen shifts ploidy in relation to its sexuality. Mol. Plant-Microbe Interact. 30: 45-52.

Li, Y., van der Lee, T. A., Evenhuis, A., van den Bosch, G. B., van Bekkum, P. J., Forch, M. G., van Gent-Pelzer, M. P., van Raaij, H. M., Jacobsen, E., Huang, S. W., et al. 2012. Population dynamics of Phytophthora infestans in the Netherlands reveals expansion and spread of dominant clonal lineages and virulence in sexual offspring. G3 (Bethesda) 2:1529-1540.

Ma, Y., Zhao, D., Yang, Z., Hu, Z., Pan, Y., Zhang, D., Xue, X., and Zhu, J. 2020. Correlation analysis of genetic composition and disease resistance of 29 potato varieties (strains) against late blight. Plant Prot. 46:212-218.

Martin, M. D., Vieira, F. G., Ho, S. Y. W., Wales, N., Schubert, M., Seguin-Orlando, A., Ristaino, J. B., and Gilbert, M. T. P. 2016. Genomic characterization of a South American Phytophthora hybrid mandates reassessment of the geographic origins of Phytophthora infestans. Mol. Biol. Evol. 33:478-491.

McDonald, B. A., and Linde, C. 2002. Pathogen population genetics, evolutionary potential, and durable resistance. Annu. Rev. Phytopathol. 40: 349-379.

McLellan, H., Boevink, P. C., Armstrong, M. R., Pritchard, L., Gomez, S., Morales, J., Whisson, S. C., Beynon, J. L., and Birch, P. R. 2013. An RxLR effector from Phytophthora infestans prevents re-localisation of two plant NAC transcription factors from the endoplasmic reticulum to the nucleus. PLoS Pathog 9:e1003670.

Na, R., Yu, D., Chapman, B. P., Zhang, Y., Kuflu, K., Austin, R., Qutob, D., Zhao, J., Wang, Y., and Gijzen, M. 2014. Genome re-sequencing and 
functional analysis places the Phytophthora sojae avirulence genes Avrlc and $e$ in a tandem repeat at a single locus. PLoS One 9:e89738.

Oh, S. K., Young, C., Lee, M., Oliva, R., Bozkurt, T. O., Cano, L. M., Win, J., Bos, J. I. B., Liu, H. Y., van Damme, M., et al. 2009. In planta expression screens of Phytophthora infestans RXLR effectors reveal diverse phenotypes, including activation of the Solanum bulbocastanum disease resistance protein Rpi-blb2. Plant Cell 21:2928-2947.

Oliva, R. F., Cano, L. M., Raffaele, S., Win, J., Bozkurt, T. O., Belhaj, K., Oh, S. K., Thines, M., and Kamoun, S. 2015. A recent expansion of the RXLR effector gene Avrblb2 is maintained in global populations of Phytophthora infestans indicating different contributions to virulence. Mol. Plant-Microbe Interact. 28:901-912.

Pais, M., Yoshida, K., Giannakopoulou, A., Pel, M. A., Cano, L. M., Oliva, R. F., Witek, K., Lindqvist-Kreuze, H., Vleeshouwers, V. G. A. A., and Kamoun, S. 2018. Gene expression polymorphism underpins evasion of host immunity in an asexual lineage of the Irish potato famine pathogen. BMC Evol. Biol. 18:93.

Qi, J. H., Asano, T., Jinno, M., Matsui, K., Atsumi, K., Sakagami, Y., and Ojika, M. 2005. Characterization of a Phytophthora mating hormone. Science 309:1828.

Qutob, D., Chapman, B. P., and Gijzen, M. 2013. Transgenerational gene silencing causes gain of virulence in a plant pathogen. Nat. Commun. 4:1349.

Raffaele, S., Farrer, R. A., Cano, L. M., Studholme, D. J., MacLean, D., Thines, M., Jiang, R. H., Zody, M. C., Kunjeti, S. G., Donofrio, N. M., et al. 2010a. Genome evolution following host jumps in the Irish potato famine pathogen lineage. Science 330:1540-1543.

Raffaele, S., Win, J., Cano, L. M., and Kamoun, S. 2010b. Analyses of genome architecture and gene expression reveal novel candidate virulence factors in the secretome of Phytophthora infestans. BMC Genomics 11:637.

Rietman, H. 2011. Putting the Phytophthora infestans genome sequence at work; multiple novel avirulence and potato resistance gene candidates revealed. Ph.D. Thesis. Wageningen University.

Saunders, D. G. O., Breen, S., Win, J., Schornack, S., Hein, I., Bozkurt, T. O., Champouret, N., Vleeshouwers, V. G. A. A., Birch, P. R. J., Gilroy, E. M., et al. 2012. Host protein BSL1 associates with Phytophthora infestans RXLR effector AVR2 and the Solanum demissum immune receptor R2 to mediate disease resistance. Plant Cell 24:3420-3434.

Shu, Y. 2015. A preliminary study in population genetic structure of Phytophthora infestans isolates collected from Anhui Fujian and Hunan provinces in China. Master Thesis. Nanjing Agricultural University. http:// oversea.cnki.net/index/

Thilliez, G. J. A., Armstrong, M. R., Lim, T. Y., Baker, K., Jouet, A., Ward, B., van Oosterhout, C., Jones, J. D. G., Huitema, E., Birch, P. R. J., et al. 2019. Pathogen enrichment sequencing (PenSeq) enables population genomic studies in oomycetes. New Phytol. 221:1634-1648.
Turnbull, D., Wang, H. X., Breen, S., Malec, M., Naqvi, S., Yang, L. N., Welsh, L., Hemsley, P., Tian, Z. D., Brunner, F., et al. 2019. AVR2 targets BSL family members, which act as susceptibility factors to suppress host immunity. Plant Physiol. 180:571-581.

van Poppel, P. M. J. A., Guo, J., de Vondervoort, P. J. I. V., Jung, M. W. M., Birch, P. R. J., Whisson, S. C., and Govers, F. 2008. The Phytophthora infestans avirulence gene Avr4 encodes an RXLR-dEER effector. Mol. Plant-Microbe Interact. 21:1460-1470.

van West, P., Shepherd, S. J., Walker, C. A., Li, S., Appiah, A. A., Grenville-Briggs, L. J., Govers, F., and Gow, N. A. R. 2008. Internuclear gene silencing in Phytophthora infestans is established through chromatin remodelling. Microbiol-Sgm 154:1482-1490.

Vleeshouwers, V. G. A. A., Raffaele, S., Vossen, J. H., Champouret, N., Oliva, R., Segretin, M. E., Rietman, H., Cano, L. M., Lokossou, A., Kessel, G., et al. 2011. Understanding and exploiting late blight resistance in the age of effectors. Annu. Rev. Phytopathol. 49:507-531.

Wang, L., Chen, H., Li, J., Shu, H., Zhang, X., Wang, Y., Tyler, B. M., and Dong, S. 2019. Effector gene silencing mediated by histone methylation underpins host adaptation in an oomycete plant pathogen. Nucleic Acids Res. 48:1790-1799.

Xiao-Hu, X., Zhi-Hui, Y., Jie-Hua, Z., Wei-Hong, Z., and Chun-Yi, W. 2013. Genetic diversity of the superphysiological race in Phytophthora infestans. Mycosystema 32:791-801.

Yang, Y. L., Hu, X. Q., Lu, S. F., Luo, W. F., Ryu, K. Y., and Xiao, L. T. 2007. Composition and distribution of physiological race of Phytophthora infestans in Yunnan Province. J. Huazhong Agric. Univ. 26:297-301.

Yin, J., Gu, B., Huang, G., Tian, Y., Quan, J., Lindqvist-Kreuze, H., and Shan, W. 2017. Conserved RXLR effector genes of Phytophthora infestans expressed at the early stage of potato infection are suppressive to host defense. Front. Plant Sci. 8:2155

Yoshida, K., Schuenemann, V. J., Cano, L. M., Pais, M., Mishra, B., Sharma, R., Lanz, C., Martin, F. N., Kamoun, S., Krause, J., et al. 2013. The rise and fall of the Phytophthora infestans lineage that triggered the Irish potato famine. eLife 2:e00731.

Zhu, S., Vossen, J. H., Bergervoet, M., Nijenhuis, M., Kodde, L., Kessel, G. J. T., Vleeshouwers, V., Visser, R. G. F., and Jacobsen, E. 2014. An updated conventional and a novel GM potato late blight $\mathrm{R}$ gene differential set for virulence monitoring of Phytophthora infestans. Euphytica 202: 219-234.

Zhu, W., Shen, L. L., Fang, Z. G., Yang, L. N., Zhang, J. F., Sun, D. L., and Zhan, J. 2016. Increased frequency of self-fertile isolates in Phytophthora infestans may attribute to their higher fitness relative to the A1 isolates. Sci. Rep. 6:29428. 